\title{
Twisted waves in symmetric and asymmetric bi-ion Kappa-distributed plasmas.
}

\author{
Kashif Arshad1, 2, a) and S. Poedts ${ }^{2,3}$ \\ 1) Department of Physics and Astronomy, University of Iowa, 52242 Iowa, USA. \\ ${ }^{2)}$ Centre for Plasma Astrophysics, Celestijnenlaan 200B, 3001 Leuven, Belgium. \\ ${ }^{3)}$ Institute of Physics, University of Maria Curie-Sktodowska, ul. Radziszewskiego 10, PL-20-031 Lublin, \\ Poland
}

(Dated: 12 November 2020)

Waves in bi-ion plasmas are affected by asymmetry. The kinetic theory of the Maxwellian and Lorentzian/Kappa-distributed bi-ion plasma is ameliorated to incorporate the transfer of orbital angular momentum from the helical electric field to the plasma modes. By operating the Laguerre-Gaussian (LG) function, the perturbed distribution function and helical electric field are decomposed into characteristic axial and azimuthal components. In symmetric bi-ion plasmas, the conventional ion modes/waves are only present if both ions have similar masses and the concentration of the electrons is negligible. An imbalance of the symmetry is considered by the contamination of a small fraction of the heavy immobile ions, which urges the negative ions to become heavier than the positive ions in the bi-ion plasma system. The distinct masses of the positive and negative ions provoke mass-asymmetry in the Kappa-distributed bi-ion plasmas. The signature of the unique acoustic-laden twisted modes in non-Maxwellian asymmetric bi-ion plasma is perceived by the temperature of the lighter positive ions and the dynamics of the heavier negative ion. The deliberated results of Landau damping are displayed for distinct values of the azimuthal wave-number and spectral index, temperature-variation and mass-asymmetry.

\section{INTRODUCTION}

Bi-ion (BI) plasmas may comprise a pair of either similar or distinct ions. In other words, bi-ion plasmas can be "symmetric" or "asymmetric". The asymmetry can concern various physical parameters like mass, temperature, density, field, potential, etc. Symmetric BI plasmas contain pairs of ions with similar masses, while their charges are equal in magnitude but of opposite sign, like for pair-ion (PI) plasma ${ }^{1}$. On the other hand, BI plasmas become asymmetric by a slight contagion, e.g. by a small fraction of high mass immobile ions. This symmetry breaking diversifies the configuration of the negative ions so that the negative ions become mildly heavier than the positive ions.

The contaminated BI plasmas may prescribe a new state of plasma. As a matter of fact, the broken symmetry can cluster the electromagnetic radiations with surplus density in the region of localization ${ }^{2}$. The temperature asymmetry is reported to be responsible for some localized stable flat top like structures due to possible nonlinear phenomena ${ }^{3}$. The examination of such predictions in controlled laboratory experiments can replicate the cosmic plasma of the hot MeV-era of the universe when it consisted primarily of electrons and positrons with a small concentration of symmetry-breaking ions ${ }^{4-6}$. Laboratory space physics research provides substantial new insights via the contribution of complementary experiments towards some of the paradigmatic phenomena tak-

a)Electronic mail: kashif.arshad.butt@gmail.com; kashif-arshad@uiowa.edu ing place in the solar corona, solar wind, planetary magnetospheres, and the outer boundary of the heliosphere ${ }^{7}$.

The pioneering kinetic theory of non-Maxwellian twisted modes was extended with the study of the Langmuir twisted modes with Kappa-distributed electrons. A further advancement was invoked with the inclusion of the Maxwellian-distributed ions/protons to investigate acoustic twisted modes carrying orbital angular momentum $^{8}$. The authors also obtained an 'exact' numerical solution for the Langmuir and acoustic twisted modes. The numerical results were found more appropriate than the analytical results, especially in the strong damping cases. Later, the twisted kinetic theory was explored for Kappa-distributed dusty plasmas ${ }^{9}$. The Dust-Ion-Acoustic and Dust-Acoustic twisted modes were modeled to examine the validity of the results depending on the electrons drift velocity, temperature of the electrons and the ions, dust charging and azimuthal wave number. (Ali et al. [2016] $)^{10}$ considered the Landau damping of twisted dusty plasma modes in the Maxwellian environment. A few years ago, the possibility of a longitudinal acoustic mode was predicted for asymmetric pair-ion plasma ${ }^{11}$. A comparable effort was made to investigate acoustic modes in dust-bi-ion electron (DBIE) plasma with heavier negative ions ${ }^{12}$.

Recently, some twisted modes such as magnetic and Alfvénic tornadoes ${ }^{13}$ have been investigated by considering non-planar (helical) fields carrying orbital angular momentum (OAM). Also, a relativistic twisted theory ${ }^{14}$ has been established for an electron wave packet by using the Dirac equation. The above studies were motivated by various applications of orbital angular momentum (OAM) such as ultra intense twisted laser pulses $^{15}$, microscopy and imaging ${ }^{16}$, ultra-fast optical 
communication $^{17}$, twisted gravitational waves ${ }^{18}$, ionospheric radar facilities ${ }^{19}$, the quantum entanglement of two photons ${ }^{20}$, Bose Einstein condensation ${ }^{21}$, and applications in astrophysics $^{22}$.

In the present manuscript, the kinetic theory is applied to the study of twisted modes in both symmetric and asymmetric BI plasmas. The Maxwellian and Kappa-distributions are considered for the symmetric BI plasma cases, while only a Kappa-distribution is chosen for asymmetric BI plasma. Non-planar twisted ion waves are studied in symmetric BI plasmas in the presence of an electric field carrying orbital angular momentum. But acoustic-type twisted ion waves are evaluated in the asymmetric BI plasmas which are contaminated by a fraction of immobile heavy ions. The three-dimensional Maxwellian distribution function can be written as;

$$
f_{0_{\alpha}}=\frac{n_{0_{\alpha}}}{(2 \pi)^{3 / 2} v_{T_{\alpha}}^{3}} \exp \left(-\frac{1}{2}\left[\frac{v_{z}^{2}}{v_{T_{\alpha}}^{2}}+\frac{v_{r}^{2}+v_{\theta}^{2}}{v_{T_{\alpha}}^{2}}\right]\right),
$$

where $T_{\alpha}$ is the ion temperature in energy units and $v_{T_{\alpha}}=\sqrt{T_{\alpha} / m}$ denotes the thermal velocity. The threedimensional Kappa(non-Maxwellian)-distribution function is defined $\mathrm{as}^{24}$;

$$
\begin{aligned}
f_{0 \alpha}= & \frac{n_{0_{\alpha}}}{\pi^{3 / 2} \theta_{\alpha}^{3}} \frac{\Gamma\left(\kappa_{\alpha}+1\right)}{\kappa_{\alpha}^{3 / 2} \Gamma\left(\kappa_{\alpha}-1 / 2\right)} \\
& \times\left[1+\frac{v_{z}^{2}}{\kappa_{\alpha} \theta_{\alpha}^{2}}+\frac{v_{r}^{2}+v_{\theta}^{2}}{\kappa_{\alpha} \theta_{\alpha}^{2}}\right]^{-\kappa_{\alpha}-1},
\end{aligned}
$$

where $\kappa_{\alpha}$ is the spectral index such that $\kappa_{\alpha}>3 / 2$ and $\theta_{\alpha}=v_{T_{\alpha}}\left(2 \kappa_{\alpha}-3\right) / \kappa_{\alpha}$ is the Kappa-distributed thermal velocity.

The remainder of this paper contains first a section on twisted waves in Maxwellian distributed symmetric biion (BI) plasma (Section II). Section III is devoted to the same kind of waves but in Kappa-distributed symmetric bi-ion (BI) plasma. In Section IV we consider the effect of asymmetry in the masses of the positive and negative ions. The obtained damping rates of the acoustic-laden twisted ion waves are subjected to a quantitative analysis in Section V. Finally, in the last section we formulate the conclusions we can draw from the present study.

\section{TWISTED WAVES IN MAXWELLIAN DISTRIBUTED SYMMETRIC BI-ION (BI) PLASMA}

This section contains a study of plasmas containing Maxwellian distributed positive and negative ions. These ions inherit similar masses, but are symmetric in terms of their opposite charges. The dielectric function $\left(\epsilon\left(\omega, k, l q_{\theta}\right)=1+\chi\right)$ of such a plasma system can be expressed as $^{9}$

$\epsilon\left(k, l q_{\theta}, \omega\right)=1+\sum_{\alpha} \frac{2 \omega_{p_{\alpha}}^{2}}{k^{2} v_{T_{\alpha}}^{2}}\left[2+\zeta_{z_{\alpha}} Z\left(\zeta_{z_{\alpha}}\right)+\zeta_{\theta_{\alpha}} Z\left(\zeta_{\theta_{\alpha}}\right)\right]$, where $\alpha= \pm$ for the lighter positive ions and heavier negative ions. The notations of the arguments $\zeta_{z_{\alpha}}=\omega / k v_{T_{\alpha}}$ and $\zeta_{\theta_{\alpha}}=\omega / l q_{\theta} v_{T_{\alpha}}$ represent their relevance to the axial and azimuthal components of the plasma dispersion function. After expanding the axial and azimuthal components of plasma dispersion functions such that $\left[\zeta_{z_{\alpha}} \gg 1\right.$ $\left.\& \zeta_{\theta_{\alpha}} \gg 1\right]$, we can derive the plasma susceptibility $\chi_{\alpha}\left(k, l q_{\theta}, \omega\right)$ of the symmetric BI plasma $\operatorname{as}^{9}$;

$$
\begin{aligned}
\chi & =\sum_{\alpha}\left[-\frac{\omega_{p_{\alpha}}^{2}}{\omega^{2}}\left[1+\frac{3 k^{2} v_{T_{\alpha}}^{2}}{\omega^{2}}\right]\right. \\
& -\frac{\omega_{p_{\alpha}}^{2}}{\varpi^{2} \omega^{2}}\left[1+\frac{3 k^{2} v_{T_{\alpha}}^{2}}{\varpi^{2} \omega^{2}}\right] \\
& +2 i \sqrt{\pi} \frac{\omega \omega_{p_{\alpha}}^{2}}{k^{3} v_{T_{\alpha}}^{3}}\left(\exp \left[-\frac{\omega^{2}}{2 k^{2} v_{T_{\alpha}}^{2}}\right]\right. \\
& \left.\left.+\varpi \exp \left[-\frac{\varpi^{2} \omega^{2}}{2 k^{2} v_{T_{\alpha}}^{2}}\right]\right)\right] .
\end{aligned}
$$

Here, $\varpi=k / l q_{\theta}$ denotes the ratio of the conventional wave number to the azimuthal wave number. Individually, the quantity $l$ imitates the number of the intertwined helices relative to the helical phase fronts of the waves/modes depending on the magnitude and the sign of $l$ as shown in Figure 1.4 of (Arshad [2018]) ${ }^{25}$, and the term $q_{\theta}$ is defined as $q_{\theta}=\int_{0}^{\infty} F_{p l}^{2} d r$. The function $F_{p l}$ is known as the Laguerre-Gaussian (LG) function and is described in the Appendix A of (Arshad et al. $[2017])^{9}$. The subscripts $p$ and $l$ represent the radial and azimuthal mode numbers, respectively. The dielectric function $\epsilon\left(\omega, k, l q_{\theta}\right)$ consists of a real $\operatorname{Re}\left[\epsilon\left(\omega, k, l q_{\theta}\right)\right]$ and an imaginary $\operatorname{Im}\left[\epsilon\left(\omega, k, l q_{\theta}\right)\right]$ part such that $\epsilon\left(\omega, k, l q_{\theta}\right)=$ $\Re\left[\epsilon\left(\omega, k, l q_{\theta}\right)\right]+i \Im\left[\epsilon\left(\omega, k, l q_{\theta}\right)\right]$ and the temporal angular frequency of the wave as $\omega=\omega_{r}+i \gamma$. The real part $\omega_{r}$ of the temporal angular frequency of the given twisted ion wave can be calculated by putting the real part of the dielectric function $\epsilon\left(\omega, k, l q_{\theta}\right)$ to zero which yields

$$
\omega_{r}^{2}=2 \omega_{p_{+}}^{2}\left(\frac{1+\varpi^{2}}{\varpi^{2}}\right)+\frac{3 k^{2} v_{\text {Teff }}^{2}}{2 \varpi^{2} \omega_{p_{+}}^{2}}\left(\frac{1+\varpi^{4}}{1+\varpi^{2}}\right)
$$

where $\omega_{p_{+}}=\omega_{p_{-}}=\sqrt{4 \pi n_{0 \pm} e^{2} / m_{ \pm}}$, is the plasma frequency of the ion species and $v_{\text {Teff }}$ is defined as

$$
v_{\mathrm{Teff}}=\omega_{p_{+}} v_{T_{+}}\left(1+\frac{T_{-}}{T_{+}}\right)^{1 / 2} .
$$

Here, $T_{+}$and $T_{-}$denote the temperatures of the positive and negative ions, while $v_{T_{+}}=\sqrt{T_{+} / m_{+}}$, is the thermal velocity of the positive ions. By using Cauchy's relation i.e, $\gamma=-\Im[\epsilon(\omega, k)] /\{\partial \Re[\epsilon(\omega, k)] / \partial \omega\}$, we can calculate the Landau damping rate $\gamma$ of the Maxwellian distributed ion waves. The twisted damping rate $\gamma$ of the Maxwellian-distributed symmetric bi-ion (BI) plasma is attained with the weak damping approximation ${ }^{27}$. Therefore, the weak damping rates should always be small (approaching zero) in comparison to the frequency 
i.e., $(\gamma \ll \omega)$ to keep the validity of the plasma dispersion solution. This weak damping approximation is implemented in the Taylor series expansion of the plasma dispersion relation, which is later integrated by using the Plemelj relation and the twisted plasma dielectric function is thus accomplished ${ }^{28}$. The weak damping rates are calculated at the phase velocity of the twisted wave and are proportional to the slope of the reduced distribution, as shown in Figure 8.11 of (Gurnett-Bhattacharjee $[2005])^{27}$. This yields

$$
\gamma=-\sqrt{\pi} \frac{\omega_{r}^{4}}{\omega_{p_{+}}^{2}}\left(\frac{\varpi^{2}}{1+\varpi^{2}}\right)\left[\gamma_{+}+\gamma_{-}\right]
$$

where

$\gamma_{+}=\frac{1}{\omega_{p_{+}} k^{3} \lambda_{D_{+}}^{3}}\left[\exp \left(-\frac{\omega_{r}^{2}}{2 k^{2} v_{T_{+}}^{2}}\right)+\varpi \exp \left(-\frac{\varpi^{2} \omega_{r}^{2}}{2 k^{2} v_{T_{+}}^{2}}\right)\right]$

and

$\gamma_{-}=\frac{1}{\omega_{p_{-}} k^{3} \lambda_{D_{-}}^{3}}\left[\exp \left(-\frac{\omega_{r}^{2}}{2 k^{2} v_{T_{-}}^{2}}\right)+\varpi \exp \left(-\frac{\varpi^{2} \omega_{r}^{2}}{2 k^{2} v_{T_{-}}^{2}}\right)\right]$

Here, $\quad \lambda_{D \pm}=\sqrt{T_{ \pm} / 4 \pi n_{0 \pm} e^{2}}$ denote the species Debye lengths. The damping rates of the studied Kappa-distributed symmetric and asymmetric BI plasmas are also calculated by employing the weak damping approximation. By using the real frequency $\omega_{r}$ given in Eq. (5) into the set of Eqs. (7)-(9), we can write the expression of the normalized damping factor as follows

$$
\begin{aligned}
\frac{\gamma}{\omega_{r}}= & -\frac{\sqrt{\pi}}{2}\left(\frac{1+\varpi^{2}}{\varpi^{2}}\right)^{1 / 2} \\
& \times \frac{1}{k^{3} \lambda_{D_{+}}^{3}}\left[\gamma_{+}+\left(\frac{T_{+}}{T_{-}}\right)^{3 / 2} \gamma_{-}\right],
\end{aligned}
$$

such that the simplified equations of the $\gamma_{+}$and $\gamma_{-}$are as follow;

$$
\begin{aligned}
\gamma_{+}= & \exp \left[-\frac{1}{2 k^{2} \lambda_{D_{+}}^{2}}\left(\frac{1+\varpi^{2}}{\varpi^{2}}\right)-\frac{1}{\varpi^{2}}\left(1+\frac{T_{-}}{T_{+}}\right)\left(\frac{1+\varpi^{4}}{1+\varpi^{2}}\right)\right] \\
& +\varpi \exp \left[-\left(\frac{1+\varpi^{2}}{2 k^{2} \lambda_{D_{+}}^{2}}\right)-\left(1+\frac{T_{-}}{T_{+}}\right)\left(\frac{1+\varpi^{4}}{1+\varpi^{2}}\right)\right],
\end{aligned}
$$

In order to calculate the dispersion relation of a nonMaxwellian Kappa distributed bi-ion plasma in the presence of non-planar (helical) electric field carrying orbital angular momentum, we will equate the real part

$$
\begin{aligned}
\gamma_{-}= & \exp \left[-\frac{1}{2 k^{2} \lambda_{D_{+}}^{2}}\left(\frac{1+\varpi^{2}}{\varpi^{2}}\right)\left(\frac{T_{+}}{T_{-}}\right)\right. \\
& \left.-\frac{1}{\varpi^{2}}\left(1+\frac{T_{-}}{T_{+}}\right)\left(\frac{1+\varpi^{4}}{1+\varpi^{2}}\right)\left(\frac{T_{+}}{T_{-}}\right)\right] \\
& +\varpi \exp \left[-\left(\frac{1+\varpi^{2}}{2 k^{2} \lambda_{D_{+}}^{2}}\right)\left(\frac{T_{+}}{T_{-}}\right)\right. \\
& \left.-\left(1+\frac{T_{-}}{T_{+}}\right)\left(\frac{1+\varpi^{4}}{1+\varpi^{2}}\right)\left(\frac{T_{+}}{T_{-}}\right)\right] .
\end{aligned}
$$

The above set of expressions concern the normalized Landau damping rates of Maxwellian-distributed twisted ion waves.

\section{TWISTED WAVES IN KAPPA-DISTRIBUTED SYMMETRIC BI-ION (BI) PLASMA}

The present section also concerns the study of plasma containing positive and negative ions with the same masses (i.e., $m_{+}=m_{-}$) and opposite charges. However, we now consider the case where the only difference between the two ion populations lies in the distribution of the particles. In fact, we here consider the distribution of the ions to be non-Maxwellian and, more precisely, 'Kappa distributed'.

$\epsilon\left(k, l q_{\theta}, \omega\right)=1+\sum_{\alpha} \frac{2 \omega_{p_{\alpha}}^{2}}{k^{2} \theta_{\alpha}^{2}}\left[\frac{2 \kappa_{\alpha}-1}{\kappa_{\alpha}}+\zeta_{z_{\alpha}} Z\left(\zeta_{z_{\alpha}}\right)+\zeta_{\theta_{\alpha}} Z\left(\zeta_{\theta_{\alpha}}\right)\right]$.

Here $\zeta_{z_{\alpha}}=\omega / k \theta_{\alpha}$ and $\zeta_{\theta_{\alpha}}=\omega / l q_{\theta} \theta_{\alpha}$ denote the axial and azimuthal arguments of the plasma dispersion function. After expansion of the plasma dispersion functions in the limit $\left[\zeta_{z_{\alpha}} \gg 1 \& \zeta_{\theta_{\alpha}} \gg 1\right]$, we can calculate the following plasma susceptibility of the Kappa-distributed symmetric BI plasma ${ }^{9}$;

$$
\begin{aligned}
\chi= & \sum_{\alpha}\left[-\frac{\omega_{p_{\alpha}}^{2}}{\omega^{2}}\left[1+\frac{3 k^{2} \theta_{\alpha}^{2}}{\omega^{2}} \frac{3 \kappa_{\alpha}}{\left(2 \kappa_{\alpha}-3\right)}\right]\right. \\
& -\frac{\omega_{p_{\alpha}}^{2}}{\varpi^{2} \omega^{2}}\left[1+\frac{3 k^{2} \theta_{\alpha}^{2}}{\varpi^{2} \omega^{2}} \frac{3 \kappa_{\alpha}}{\left(2 \kappa_{\alpha}-3\right)}\right] \\
& +2 i \sqrt{\pi} \frac{\omega \Gamma\left(\kappa_{\alpha}+1\right)}{\kappa_{\alpha}^{3 / 2} \Gamma\left(\kappa_{\alpha}-1 / 2\right)} \frac{\omega_{p e}^{2}}{k^{3} \theta_{\alpha}^{3}} \\
& \times\left(\left[1+\frac{\omega^{2}}{\kappa_{\alpha} k^{2} \theta_{\alpha}^{2}}\right]^{-\kappa_{\alpha}-1}\right. \\
& \left.\left.+\varpi\left[1+\varpi^{2} \frac{\omega^{2}}{\kappa_{\alpha} k^{2} \theta_{\alpha}^{2}}\right]^{-\kappa_{\alpha}-1}\right)\right] .
\end{aligned}
$$


$\Re\left[\epsilon\left(\omega, k, l q_{\theta}\right)\right]$ of the dielectric function to zero, yielding

$$
\begin{aligned}
\omega_{r}^{2}= & \omega_{p_{+}}^{2}\left(\frac{1+\varpi^{2}}{\varpi^{2}}\right)+\frac{3 \kappa_{+}}{\left(2 \kappa_{+}-3\right)} \\
& \times \frac{k^{2} \theta_{T e f f}^{2}}{2 \varpi^{2} \omega_{p_{+}}^{2}}\left(\frac{1+\varpi^{4}}{1+\varpi^{2}}\right),
\end{aligned}
$$

where

$$
\theta_{\mathrm{Teff}}=\omega_{p_{+}} \theta_{+}\left(1+\frac{\kappa_{-}\left(2 \kappa_{+}-3\right)}{\kappa_{+}\left(2 \kappa_{-}-3\right)} \frac{T_{-}}{T_{+}}\right)^{1 / 2}
$$

By using Cauchy's relation again, i.e. $\gamma=$ $-\Im[\epsilon(\omega, k)] / \partial \Re[\epsilon(\omega, k)] / \partial \omega$, we can calculate the following expression for the damping rate $\gamma$ :

$$
\gamma=-\sqrt{\pi} \frac{\omega_{r}^{4}}{\omega_{p_{+}}^{2}}\left(\frac{\varpi^{2}}{1+\varpi^{2}}\right)\left[\gamma_{+}+\gamma_{-}\right]
$$

Here

$$
\begin{aligned}
\gamma_{+}= & \frac{1}{\omega_{p_{+}} k^{3} \lambda_{D_{+}}^{3}} \frac{\Gamma\left(\kappa_{+}+1\right)}{\kappa_{+}^{3 / 2} \Gamma\left(\kappa_{+}-\frac{1}{2}\right)}\left[\left\{1+\frac{\left(\omega_{r} / k\right)^{2}}{\kappa_{+} \theta_{+}^{2}}\right\}^{-\kappa_{+}-1}\right. \\
& \left.+\varpi\left\{1+\frac{\varpi^{2}\left(\omega_{r} / k\right)^{2}}{\kappa_{+} \theta_{+}^{2}}\right\}^{-\kappa_{+}-1}\right],
\end{aligned}
$$

and

$$
\begin{aligned}
\gamma_{-}= & \frac{1}{\omega_{p_{-}} k^{3} \lambda_{D_{-}}^{3}} \frac{\Gamma\left(\kappa_{-}+1\right)}{\kappa_{-}^{3 / 2} \Gamma\left(\kappa_{-}-\frac{1}{2}\right)}\left[\left\{1+\frac{\left(\omega_{r} / k\right)^{2}}{\kappa_{-} \theta_{-}^{2}}\right\}^{-\kappa_{-}-1}\right. \\
& \left.+\varpi\left\{1+\frac{\varpi^{2}\left(\omega_{r} / k\right)^{2}}{\kappa_{-} \theta_{-}^{2}}\right\}^{-\kappa_{-}-1}\right] .
\end{aligned}
$$

By substituting the real part of the plasma dispersion relation i.e., $\omega_{r}$ from Eq. (15) into the set of damping Eqs. (17) -(19), the normalized damping factor is then given by

$$
\begin{aligned}
\frac{\gamma}{\omega_{r}}= & -\frac{\sqrt{\pi}}{2}\left(\frac{1+\varpi^{2}}{\varpi^{2}}\right)^{1 / 2} \\
& \frac{1}{k^{3} \lambda_{D_{+}}^{3}}\left[\gamma_{+}+\left(\frac{T_{+}}{T_{-}}\right)^{3 / 2} \gamma_{-}\right],
\end{aligned}
$$

where the simple mathematical forms of $\gamma_{+}$and $\gamma_{-}$are expressed in the following manner;

$$
\begin{aligned}
\gamma_{+} & =\frac{\Gamma\left(\kappa_{+}+1\right)}{\left(2 \kappa_{+}-3\right)^{3 / 2} \Gamma\left(\kappa_{+}-\frac{1}{2}\right)} \\
& \times\left[\left\{1+\frac{1}{\left(2 \kappa_{+}-3\right) k^{2} \lambda_{D_{+}}^{2}}\left(\frac{1+\varpi^{2}}{\varpi^{2}}\right)+\frac{1}{\varpi^{2}}\right.\right. \\
& \left.\times \frac{3}{\left(2 \kappa_{+}-3\right)}\left(1+\frac{\kappa_{-}\left(2 \kappa_{+}-3\right)}{\kappa_{+}\left(2 \kappa_{-}-3\right)} \frac{T_{-}}{T_{+}}\right)\left(\frac{1+\varpi^{4}}{1+\varpi^{2}}\right)\right\} \\
& +\varpi\left\{1+\frac{1+\varpi^{2}}{\left(2 \kappa_{+}-3\right) k^{2} \lambda_{D_{+}}^{2}}+\frac{3}{\left(2 \kappa_{+}-3\right)}\right. \\
& \left.\left.\times\left(1+\frac{\kappa_{-}\left(2 \kappa_{+}-3\right)}{\kappa_{+}\left(2 \kappa_{-}-3\right)} \frac{T_{-}}{T_{+}}\right)\left(\frac{1+\varpi^{4}}{1+\varpi^{2}}\right)\right\}^{-\kappa_{+}-1}\right],
\end{aligned}
$$

and

$$
\begin{aligned}
\gamma_{-} & =\frac{\Gamma\left(\kappa_{-}+1\right)}{\kappa_{-}^{3 / 2} \Gamma\left(\kappa_{-}-\frac{1}{2}\right)} \\
& \times\left[\left\{1+\left(\frac{T_{+}}{T_{-}}\right) \frac{1}{\left(2 \kappa_{-}-3\right) k^{2} \lambda_{D_{+}}^{2}}\right.\right. \\
& \times\left(\frac{1+\varpi^{2}}{\varpi^{2}}\right)+\frac{1}{\varpi^{2}} \frac{3}{\left(2 \kappa_{-}-3\right)}\left(\frac{T_{+}}{T_{-}}\right) \\
& \left.\times\left(1+\frac{\kappa_{-}\left(2 \kappa_{+}-3\right)}{\kappa_{+}\left(2 \kappa_{-}-3\right)} \frac{T_{-}}{T_{+}}\right)\left(\frac{1+\varpi^{4}}{1+\varpi^{2}}\right)\right\}^{-\kappa_{-}-1} \\
& +\varpi\left\{1+\left(\frac{T_{+}}{T_{-}}\right) \frac{\left(1+\varpi^{2}\right)}{\left(2 \kappa_{-}-3\right) k^{2} \lambda_{D_{+}}^{2}}+\frac{3}{\left(2 \kappa_{+}-3\right)}\right. \\
& \left.\left.\times\left(\frac{T_{+}}{T_{-}}\right)\left(1+\frac{\kappa_{-}\left(2 \kappa_{+}-3\right)}{\kappa_{+}\left(2 \kappa_{-}-3\right)} \frac{T_{-}}{T_{+}}\right)\left(\frac{1+\varpi^{4}}{1+\varpi^{2}}\right)\right\}^{-\kappa_{-}-1}\right] .
\end{aligned}
$$

In the limit that both $\varpi=k / l q_{\theta}$ (the ratio of the parallel to the azimuthal wave number) and the spectral index $\kappa$ are approaching to infinity, i.e. $\varpi \rightarrow \infty$ and $\kappa \rightarrow \infty$, the obtained Landau damping rate is analogous to that given by (Arshad-Mahmood [2010] $)^{1}$.

\section{TWISTED WAVES IN MAXWELLIAN DISTRIBUTED ASYMMETRIC BI-ION (BI) PLASMA}

In this section, we consider the Maxwellian distributed BI plasma containing a pair of Maxwellian distributed positive and negative ions with distinct masses (i.e., $\left.m_{+} \neq m_{-}\right)$. The negative ions are marginally heavier than the positive ions. Therefore, We can handle the Maxwellian distributed positive ions as Boltzmannian species, while the Maxwellian negative ions as inertial. This implies that the wave dynamics are well described by the temperature of the positive ions and inertia of the negative ions. In such a situation, we can write the dielectric function of the Maxwellian distributed BI plasma 
in the following manner

$$
\epsilon\left(k, l q_{\theta}, \omega\right)=1+\sum_{\alpha} \frac{2 \omega_{p_{\alpha}}^{2}}{k^{2} v_{T_{\alpha}}^{2}}\left[2+\zeta_{z_{\alpha}} Z\left(\zeta_{z_{\alpha}}\right)+\zeta_{\theta_{\alpha}} Z\left(\zeta_{\theta_{\alpha}}\right)\right],
$$

where $\zeta_{z_{\alpha}}=\omega / k v_{T_{\alpha}}$ and $\zeta_{\theta_{\alpha}}=\omega / l_{q_{\theta}} v_{T_{\alpha}}$ represent the arguments of the total plasma dispersion function, which is composed of the axial and azimuthal components. The difference in the ion masses will constitute contrasting plasma susceptibilities of the positive and negative ions. Therefore, the plasma susceptibility of the Boltzmannian positive ions can be expressed under the condition $\left[\zeta_{z_{+}} \ll\right.$ $\left.1 \& \zeta_{\theta_{+}} \gg 1\right]$ as $^{9}$

$$
\begin{aligned}
\chi_{+}= & \frac{1}{k^{2} \lambda_{D+}^{2}}+2 i \sqrt{\pi} \frac{\omega \omega_{p+}^{2}}{k^{3} \theta_{+}^{3}} \\
& \times\left[1+\varpi \exp \left(-\frac{\varpi^{2} \omega^{2}}{2 k^{2} v_{T_{+}}^{2}}\right)\right],
\end{aligned}
$$

and to appraise the contribution of massive negative ions to the wave dynamics, the plasma susceptibility of heavier negative ions comprise the limit $\left[\zeta_{z_{-}} \gg 1 \& \zeta_{\theta_{-}} \gg 1\right]$ and can be represented as

$$
\begin{aligned}
\chi_{-}= & -\frac{\omega_{p_{-}}^{2}}{\omega^{2}}\left[1+\frac{3 k^{2} v_{T_{-}}^{2}}{\omega^{2}}\right] \\
& -\frac{\omega_{p_{-}}^{2}}{\varpi^{2} \omega^{2}}\left[1+\frac{3 k^{2} v_{T_{-}}^{2}}{\varpi^{2} \omega^{2}}\right]+2 i \sqrt{\pi} \frac{\omega \omega_{p-}^{2}}{k^{3} v_{T_{-}}^{3}} \\
& \times\left[\exp \left(-\frac{\omega^{2}}{2 k^{2} v_{T_{-}}^{2}}\right)+\varpi \exp \left(-\frac{\varpi^{2} \omega^{2}}{2 k^{2} v_{T_{-}}^{2}}\right)\right]
\end{aligned}
$$

Again, the dispersion relation of the acoustic-type twisted ion wave can be evaluated by equating the real part of the dielectric function $\epsilon\left(\omega, k, l q_{\theta}\right)$ to zero. This now yields:

$$
\omega_{r}^{2}=\frac{k^{2}}{\varpi^{2}}\left(\frac{T_{+}}{m_{-}}\right)\left[\frac{\left(1+\varpi^{2}\right)}{\left(1+k^{2} \lambda_{D+}^{2}\right)}+\left(\frac{3 T_{-}}{T_{+}}\right) \frac{\left(1+\varpi^{4}\right)}{\left(1+\varpi^{2}\right)}\right] .
$$

The above equation displays the dispersion relation of acoustic-type twisted ion waves in Maxwellian distributed BI plasma. The Landau damping rate $\gamma$ of Maxwellian distributed acoustic-type twisted ion waves can be obtained from Cauchy's relation in the presence of helical non-planar electric field. By using $\Im(\epsilon)$ and $\Re(\epsilon)$ in Cauchy's relation, we can obtain the following relation of $\gamma$ :

$$
\gamma=-\frac{\sqrt{\pi} \omega_{r}^{3} \varpi^{2}}{k^{2} \omega_{p_{-}}^{2}\left(1+\varpi^{2}\right)}\left[\gamma_{+}+\gamma_{-}\right]
$$

where $\gamma_{+}$and $\gamma_{-}$are illustrating the contributions of the positive and negative ions to the damping factor and are defined as

$$
\gamma_{+}=\frac{\omega_{r}}{k} \frac{\omega_{p_{+}}^{2}}{v_{T_{+}}^{3}}\left[1+\varpi \exp \left(-\frac{\varpi^{2} \omega^{2}}{2 k^{2} v_{T_{+}}^{2}}\right)\right],
$$

and

$$
\begin{aligned}
\gamma_{-}= & \frac{\omega_{r}}{k} \frac{\omega_{p_{-}}^{2}}{v_{T_{-}}^{3}}\left[\exp \left(-\frac{\omega^{2}}{2 k^{2} v_{T_{-}}^{2}}\right)\right. \\
& \left.+\varpi \exp \left(-\frac{\varpi^{2} \omega^{2}}{2 k^{2} v_{T_{-}}^{2}}\right)\right] .
\end{aligned}
$$

Again substituting the real component of the plasma dispersion $\left(\omega_{r}\right)$ from Eq. (26) into the set of damping Eqs. (27)-(29), we can write the following dimensionless weak damping rate of the acoustic-laden twisted ion waves

$$
\frac{\gamma}{\omega_{r}}=-\frac{\sqrt{\pi}\left(1+\varpi^{2}\right)^{1 / 2}}{\varpi\left(1+k^{2} \lambda_{D+}^{2}\right)^{3 / 2}}\left[\sqrt{\frac{m_{+}}{m_{-}}} \gamma_{+}+\gamma_{-}\right],
$$

and the simplified forms of $\gamma_{+}$and $\gamma_{-}$are as follows

$$
\gamma_{+}=\left[1+\varpi \exp \left(-\frac{\left(1+\varpi^{2}\right)}{\left(1+k^{2} \lambda_{D+}^{2}\right)}\left(\frac{m_{+}}{m_{-}}\right)\right)\right],
$$

and

$$
\begin{aligned}
\gamma_{-}= & \left(\frac{T_{+}}{T_{-}}\right)^{3 / 2}\left[\exp \left(-\frac{\left(1+\varpi^{2}\right)}{\varpi^{2}\left(1+k^{2} \lambda_{D+}^{2}\right)}\left(\frac{T_{+}}{T_{-}}\right)\right)\right. \\
& \left.+\varpi \exp \left(-\frac{\left(1+\varpi^{2}\right)}{\left(1+k^{2} \lambda_{D+}^{2}\right)}\left(\frac{T_{+}}{T_{-}}\right)\right)\right] .
\end{aligned}
$$

In the limit that the $\varpi=k / l q_{\theta}$ (the ratio of the parallel to the azimuthal wave number) approaches to infinity, i.e. $\varpi \rightarrow \infty$, the obtained Landau damping rate is analogous to that given by (Alexandrov et al. [1984]) ${ }^{26}$.

\section{TWISTED WAVES IN KAPPA-DISTRIBUTED ASYMMETRIC BI-ION (BI) PLASMA}

In the present section, we consider the case when the Kappa distributed positive and negative ions exhibit a variation in their masses, i.e. we consider positive ions that are slightly lighter than the negative ions. Therefore, the Kappa distributed positive ions are treated as Boltzmannian, while the Kappa-distributed negative ions are inertial. This means that the temperature of the positive ions and mass of the negative ions are important to describe the dynamics of the waves. In such a scenario, we can represent the plasma dielectric function of the Kappa-distributed BI plasma containing Boltzmannian positive ions and dynamic negative ions in the following way

$\epsilon\left(k, l q_{\theta}, \omega\right)=1+\sum_{\alpha} \frac{2 \omega_{p_{\alpha}}^{2}}{k^{2} \theta_{\alpha}^{2}}\left[\frac{2 \kappa_{\alpha}-1}{\kappa_{\alpha}}+\zeta_{z_{\alpha}} Z\left(\zeta_{z_{\alpha}}\right)+\zeta_{\theta_{\alpha}} Z\left(\zeta_{\theta_{\alpha}}\right)\right]$, 
where $\zeta_{z_{\alpha}}=\omega / k \theta_{\alpha}$ and $\zeta_{\theta_{\alpha}}=\omega / l q_{\theta} \theta_{\alpha}$ are again the arguments of the total plasma dispersion function comprising axial and azimuthal components. Due to distinct masses of the pair of ions, the plasma susceptibility of the positive ions will differ from those of the negative ions. Therefore, we can write the plasma susceptibility of the lighter positive ions by applying $\left[\zeta_{z_{+}} \ll 1 \& \zeta_{\theta_{+}} \gg 1\right] \operatorname{as}^{9}$

$$
\begin{aligned}
\chi_{+}= & \frac{1}{k^{2} \lambda_{D+}^{2}} \frac{\left(\kappa_{+}-1 / 2\right)}{\left(\kappa_{+}-3 / 2\right)} \\
& +2 i \sqrt{\pi} \frac{\omega \Gamma\left(\kappa_{+}+1\right)}{\kappa_{+}^{3 / 2} \Gamma\left(\kappa_{+}-1 / 2\right)} \frac{\omega_{p+}^{2}}{k^{3} \theta_{+}^{3}} \\
& \times\left[1+\varpi\left(1+\varpi^{2} \frac{\omega^{2}}{\kappa_{+} k^{2} \theta_{+}^{2}}\right)^{-\kappa_{+}-1}\right],
\end{aligned}
$$

and quantify the role of heavier negative ions contributing to the dynamics of the plasma mode. The plasma susceptibility of negative ions comprise the limit $\left[\zeta_{z_{-}} \gg 1\right.$ $\left.\& \zeta_{\theta_{-}} \gg 1\right]$ and can be expressed as

$$
\begin{aligned}
\chi_{-}= & -\frac{\omega_{p_{-}}^{2}}{\omega^{2}}\left[1+\frac{3 k^{2} \theta_{-}^{2}}{\omega^{2}} \frac{3 \kappa_{-}}{\left(2 \kappa_{-}-3\right)}\right] \\
& -\frac{\omega_{p_{-}}^{2}}{\varpi^{2} \omega^{2}}\left[1+\frac{3 k^{2} \theta_{-}^{2}}{\varpi^{2} \omega^{2}} \frac{3 \kappa_{-}}{\left(2 \kappa_{-}-3\right)}\right] \\
& +2 i \sqrt{\pi} \frac{\omega \Gamma\left(\kappa_{-}+1\right)}{\kappa_{-}^{3 / 2} \Gamma\left(\kappa_{-}-1 / 2\right)} \frac{\omega_{p-}^{2}}{k^{3} \theta_{-}^{3}} \\
& \times\left[\left(1+\frac{\omega^{2}}{\kappa_{-} k^{2} \theta_{-}^{2}}\right)^{-\kappa_{-}-1}\right. \\
& \left.+\varpi\left(1+\varpi^{2} \frac{\omega^{2}}{\kappa_{-} k^{2} \theta_{-}^{2}}\right)^{-\kappa_{-}-1}\right] .
\end{aligned}
$$

Again, the dispersion relation of the acoustic-type ion wave can be calculated by equating the real part of the dielectric function $\epsilon\left(\omega, k, l q_{\theta}\right)$ to zero. This now yields: contributions are given by the expressions

$$
\begin{aligned}
\gamma_{+}= & \frac{\omega_{r}}{k} \frac{\omega_{p_{+}}^{2}}{\theta_{+}^{3}} \frac{\Gamma\left(\kappa_{+}+1\right)}{\kappa_{+}^{3 / 2} \Gamma\left(\kappa_{+}-1 / 2\right)} \\
& {\left[1+\varpi\left(1+\varpi^{2} \frac{\omega_{r}^{2}}{\kappa_{+} k^{2} \theta_{+}^{2}}\right)^{-\kappa_{+}-1}\right], }
\end{aligned}
$$

and

$$
\begin{aligned}
\gamma_{-}= & \frac{\omega_{r}}{k} \frac{\omega_{p_{-}}^{2}}{\theta_{-}^{3}} \frac{\Gamma\left(\kappa_{-}+1\right)}{\kappa_{-}^{3 / 2} \Gamma\left(\kappa_{-}-1 / 2\right)}\left[\left(1+\frac{\omega^{2}}{\kappa_{-} k^{2} \theta_{-}^{2}}\right)^{-\kappa_{-}-1}\right. \\
& \left.+\varpi\left(1+\varpi^{2} \frac{\omega^{2}}{\kappa_{-} k^{2} \theta_{-}^{2}}\right)^{-\kappa_{-}-1}\right] .
\end{aligned}
$$

Again inserting the real component of the plasma dispersion $\left(\omega_{r}\right)$ from Eq. (36) into the set of damping Eqs. (37)(39), we can get the following dimensionless weak damping rate of the acoustic-laden twisted ion waves

$$
\frac{\gamma}{\omega_{r}}=-\frac{\sqrt{\pi}\left(1+\varpi^{2}\right)^{1 / 2}}{\varpi\left(2 \frac{\left(\kappa_{+}-1 / 2\right)}{\left(\kappa_{+}-3 / 2\right)}+k^{2} \lambda_{D+}^{2}\right)^{3 / 2}}\left[\sqrt{\frac{m_{+}}{m_{-}}} \gamma_{+}+\gamma_{-}\right]
$$

and the simplified forms of $\gamma_{+}$and $\gamma_{-}$are as follows

$$
\begin{aligned}
\gamma_{+}= & \frac{\Gamma\left(\kappa_{-}+1\right)}{\left(2 \kappa_{-}-3\right)^{3 / 2} \Gamma\left(\kappa_{-}-1 / 2\right)}[1+\varpi \\
& \left.\times\left(1+\frac{\left(1+\varpi^{2}\right) M_{\kappa}}{\left(2 \frac{\left(\kappa_{+}-1 / 2\right)}{\left(\kappa_{+}-3 / 2\right)}+k^{2} \lambda_{D+}^{2}\right)}\right)^{-\kappa_{+}-1}\right],
\end{aligned}
$$

and

$$
\begin{aligned}
\gamma_{-}= & \left(\frac{T_{+}}{T_{-}}\right)^{3 / 2} \frac{\Gamma\left(\kappa_{-}+1\right)}{\left(2 \kappa_{-}-3\right)^{3 / 2} \Gamma\left(\kappa_{-}-1 / 2\right)} \\
& \times\left[\left(1+\frac{\left(1+\varpi^{2}\right) T_{\kappa}}{\varpi^{2}\left(2 \frac{\left(\kappa_{+}-1 / 2\right)}{\left(\kappa_{+}-3 / 2\right)}+k^{2} \lambda_{D+}^{2}\right)}\right)^{-\kappa_{-}-1}\right.
\end{aligned}
$$$$
\left.\omega_{r}^{2}=\frac{k^{2}}{\varpi^{2}}\left(\frac{T_{+}}{m_{-}}\right)\left[\frac{\left(1+\varpi^{2}\right)}{\left(2 \frac{\left(\kappa_{+}-1 / 2\right)}{\left(\kappa_{+}-3 / 2\right)}+k^{2} \lambda_{D+}^{2}\right)}+\left(\frac{3 T_{-}}{T_{+}}\right) \frac{\left(1+\varpi^{4}\right)}{\left(1+\varpi^{2}\right)}\right] \cdot \quad+\varpi\left(1+\frac{\left(1+\varpi^{2}\right) T_{\kappa}}{\left(2 \frac{\left(\kappa_{+}-1 / 2\right)}{\left(\kappa_{+}-3 / 2\right)}+k^{2} \lambda_{D+}^{2}\right)}\right)^{-\kappa_{-}-1}\right] .
$$

Where the quantity $T_{\kappa}$ is defined as $T_{\kappa}=T_{+} / T_{-}\left(2 \kappa_{-}-3\right)$ and $M_{\kappa}$ as $M_{\kappa}=m_{+} / m_{-}\left(2 \kappa_{+}-3\right)$. In the limit that both $\varpi=k / l q_{\theta}$ (the ratio of the parallel to the azimuthal wave number) and the spectral index $\kappa$ are approaching to infinity, i.e. $\varpi \rightarrow \infty$ and $\kappa \rightarrow \infty$, the obtained Landau damping rate is analogous to that given by (Alexandrov et al. $[1984])^{26}$.

$$
\gamma=-\frac{\sqrt{\pi} \omega_{r}^{3} \varpi^{2}}{k^{2} \omega_{p_{-}}^{2}\left(1+\varpi^{2}\right)}\left[\gamma_{+}+\gamma_{-}\right]
$$

where $\gamma_{+}$and $\gamma_{-}$are representing the contributions of the positive and negative ions to the damping factor. These

\section{QUANTITATIVE ANALYSIS}

In this section, the damping rates of the classical and acoustic-laden twisted ion wave are quantified for specific 


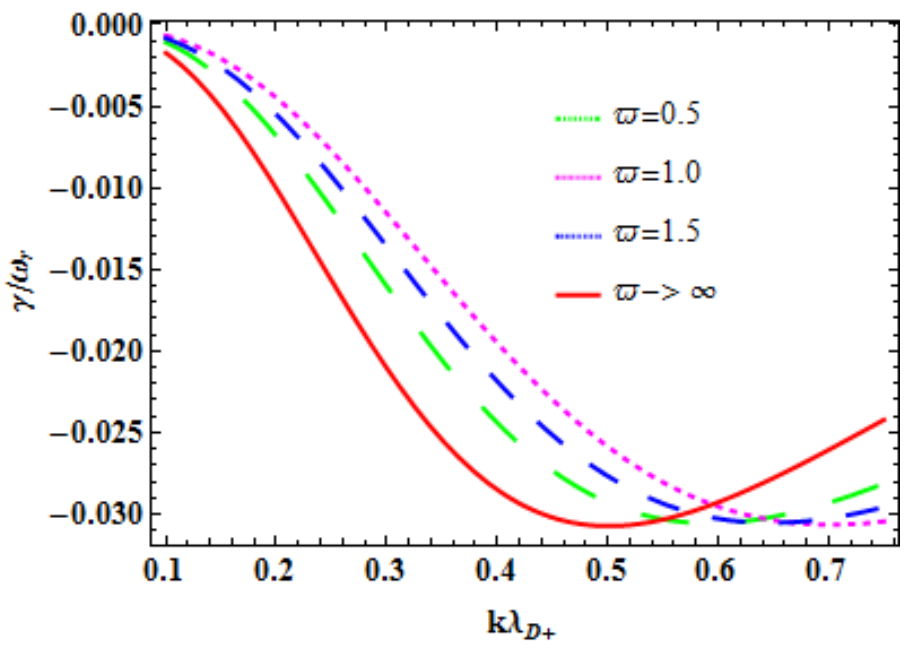

FIG. 1. The plots of normalized Landau damping rate $\gamma / \omega_{r}$ obtained analytically are presented against the normalized Debye length of positive ions $k \lambda_{D+}$, for the Kappa-distributed twisted ion modes using different values of $\varpi=k / l q_{\theta}$ at $\kappa_{+}=$ $2, \kappa_{-}=3, m_{+} / m_{-}=1$ and $T_{+} / T_{-}=40$.

parameter choices and plotted versus $k \lambda_{D+}$ (the normalized Debye length of positive ions) in bi-ion plasma containing non-thermal Kappa distributed positive and negative ions ${ }^{12}$. In Figures 1 to 10, we study the dependency of the normalized Landau damping rate $\gamma / \omega_{r}$ on various parameters. Of course, the damping rate is larger, i.e. the damping is stronger, when $\gamma / \omega_{p_{-}}$becomes more negative.

First, we will consider the twisted ion waves in a nonMaxwellian Kappa-distributed symmetric bi-ion plasma (having positive and negative ions with similar masses and distinct spectral indices), i.e. $m_{+} / m_{-}=1, \kappa_{+}=2$ and $\kappa_{-}=3$. Figure 1 shows the normalized Landau damping rate $\gamma / \omega_{r}$ the normalized Debye length of positive ions $k \lambda_{D+}$, and for different values of $\varpi=k / l q_{\theta}$ (the ratio of the parallel to the azimuthal wave number). We have included four values of $\varpi=k / l q_{\theta}$ to portray the normalized Landau damping rate of the Kappadistributed twisted ion wave, especially (i) for the case with an azimuthal wave number greater than the parallel (axial) wave number, $l q_{\theta}>k$ such that $\varpi=0.5$; (ii) the case when the azimuthal wave number is equal to the parallel wave number, $l q_{\theta}=k$ such that $\varpi=1$; and (iii) for the case where the azimuthal wave number is less than the parallel wave number, $l q_{\theta}<k$ such that $\varpi=1.5$, for a helical (azimuthal electric field), respectively, and one for the planar electric field case (iv) for a very small azimuthal wave number (so that $l q_{\theta} \cong 0$ ) in comparison to the parallel wave number $k$ such that $\varpi=\infty$.

The figure elucidates that the normalized Landau damping rate of the twisted ion wave, $\gamma / \omega_{r}$, is the smallest (in absolute value) for $\varpi=1$, while it is mildly larger for the larger $\varpi=1.5$ and considerably larger for the smaller $\varpi=0.5$, as compared to each other for the helical

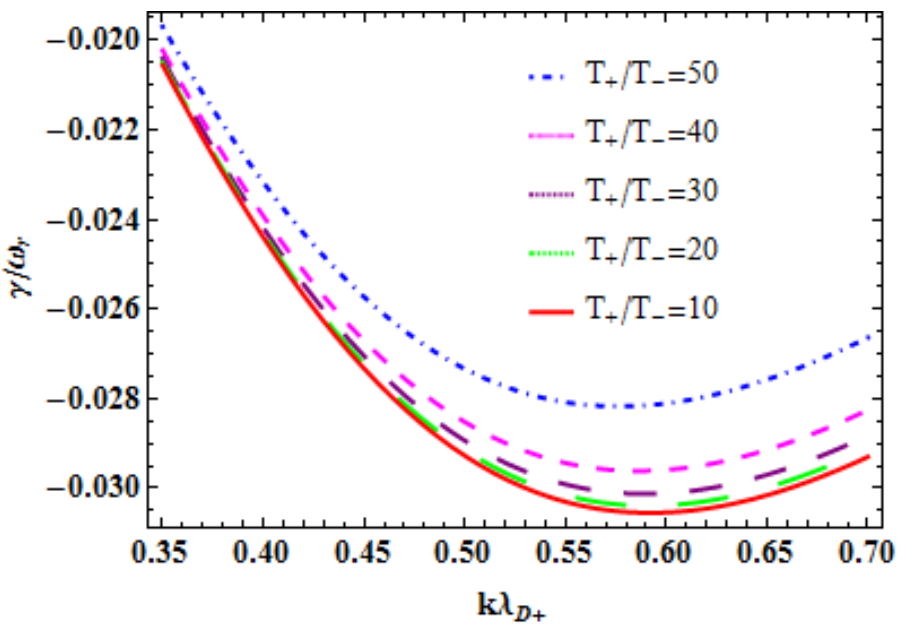

FIG. 2. The plots of normalized Landau damping rate $\gamma / \omega_{r}$ obtained analytically are presented against the normalized Debye length of positive ions $k \lambda_{D+}$, for the Kappa-distributed twisted ion modes using different values of $T_{+} / T_{-}$at $\kappa_{+}=$ $2, \kappa_{-}=3, m_{+} / m_{-}=1$ and $\varpi=0.5$.

(azimuthal electric field) cases. The scientific delineation of the largest damping rate is the presence of the large number of particles interacting with the twisted ion wave for the $\varpi=0.5<1$ case. Therefore, the damping rate of the twisted ion wave for the $\varpi=0.5<1$ case is traced larger (in absolute value) than for the cases $\varpi=1.5$ and $\varpi=1.0$. However, if we analyze the damping curves of the twisted ion wave case $(\varpi=0.5,1,1.5)$ correlative to the planar ion wave case $(\varpi=\infty)$, the red planar curve emerges below the twisted wave curves. Hence, the damping rates of non-planar twisted ion wave is smaller in comparison to the planar ion wave.

Figure 2 manifests the normalized Landau damping rates $\gamma / \omega_{p_{-}}$of the twisted ion wave in the nonMaxwellian Kappa-distributed symmetric bi-ion plasma (with similar masses and distinct spectral indices), i.e. $m_{+} / m_{-}=1, \kappa_{+}=2$ and $\kappa_{-}=3$ and such that the temperature of the positive ions is higher than that of the negative ions, i.e. $T_{+}>T_{-}$. The normalized Landau damping rate $\gamma / \omega_{p_{-}}$is plotted against the normalized Debye length of the positive ions, $k \lambda_{D+}$, for five different values of the temperature ratio of positive to negative ions viz. $T_{+} / T_{-}=10,20,30,40$ and 50 . It is evident that the normalized damping rate $\gamma / \omega_{p_{-}}$of the twisted ion wave is minimal for $T_{+} / T_{-}=10$ (corresponding to the maximal damping rate $\left.\gamma / \omega_{p_{-}}\right)$and maximal for $T_{+} / T_{-}=50$ (corresponding to the minimal damping rate). It is evinced that the Landau damping rate of the twisted ion wave increases with descending magnitude of the temperature ratio of positive to negative ions, $T_{+} / T_{-}$, and decreases with ascending temperature ratio of positive to negative ions. The reason for such behavior is again associated with the wave-particle interaction phenomenon. The supra-thermal particles can transmit more energy to the twisted ion wave, if the temperature 
ratio of positive to negative ions, $T_{+} / T_{-}$, is higher and the Landau damping rate of the twisted ion wave decreases. Therefore, the Landau damping of the twisted ion wave is higher for the smaller value of the temperature ratio of positive to negative ions, because the energy supply by the particles to the wave consequently reduces with lower $T_{+} / T_{-}$.

Now, the acoustic-laden twisted ion waves is elucidated to investigate the dispersion of the real frequency $\omega_{r}$ in Kappa-distributed asymmetric bi-ion plasma (with distinct masses and spectral indices of positive and negative ions ) i.e., $m_{+} / m_{-}=0.1, \kappa_{+}=2$ and $\kappa_{-}=3$. Figure 3 annotates the surface plot of the normalized real frequency $\omega_{r} / \omega_{p_{-}}$against the normalized Debye length of positive ions $k \lambda_{D+}$ for fixed values of the temperature ratio of positive to negative ions $T_{+} / T_{-}$and $\varpi=k / l q_{\theta}$. It can be seen that the normalized real frequency $\omega_{r} / \omega_{p_{-}}$ ascends with increasing range of the normalized Debye length of positive ions $k \lambda_{D+}$. It is obvious that the large number of suprathermal particles are probable for the larger value of the normalized Debye length of positive ions $k \lambda_{D+}$. Therefore, the immense number of suprathermal particles contribute more to magnitude of the frequency. That's why, the altitude of the curve rises with the elevation in the value of the normalized Debye length of positive ions $k \lambda_{D+}$.

Fig. 3(a) illustrates the results of the normalized real frequency $\omega_{r} / \omega_{p_{-}}$, which are produced by the analytical solution of the plasma dielectric function. Whlie the exact numerical results of the normalized real frequency $\omega_{r} / \omega_{p_{-}}$are attained Fig. 3(b) by using exact numerical solution of the plasma dielectric function.

The comparison of approximative analytical and exact numerical results (shown in the upper and lower panels, respectively) of the normalized real frequency $\omega_{r} / \omega_{p_{-}}$ has been displayed in the Fig. 3(c). It is revealed that the normalized real frequency $\omega_{r} / \omega_{p_{-}}$has higher magnitude for the numerical results and even the area under the curve is more for the numerical results. In the high frequency region, the numerical curve approaches the analytical curve, until they cut each other about $k \lambda_{D+}=0.99$. While the frequency curves exhibit the asymptotic demeanor owing to the shorter wavelength (i.e., smaller values of $k \lambda_{D+}$ ) correlative to the low frequency region. This is because that the plasma dielectric function is solved till the second order-term for obtaining the analytical results. That's why, the analytical results evince contradiction with the numerical results in the low frequency region.

Figure 4 declares the plots of the normalized real frequency $\omega_{r} / \omega_{p_{-}}$relative to the acoustic-laden twisted ion wave in Kappa-distributed asymmetric bi-ion plasma (having distinct masses and spectral indices for the positive and negative ions) i.e., $m_{+} \neq m_{-}, \kappa_{+}=2$ and $\kappa_{-}=3$. The normalized real frequency $\omega_{r} / \omega_{p_{-}}$is plotted corresponding to the temperature ratio of positive to negative ions $T_{+} / T_{-}$. It is observed that the normalized real frequency $\omega_{r} / \omega_{p_{-}}$descends with increasing value of
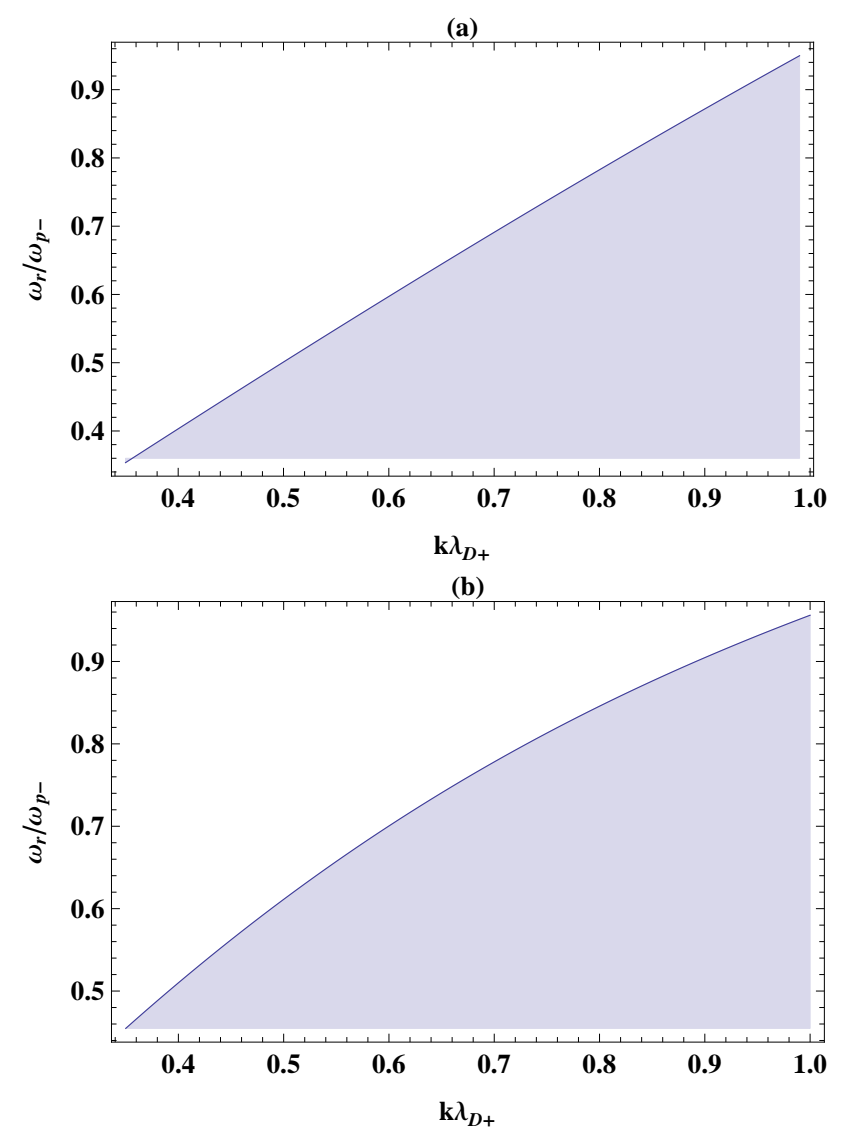

(c)

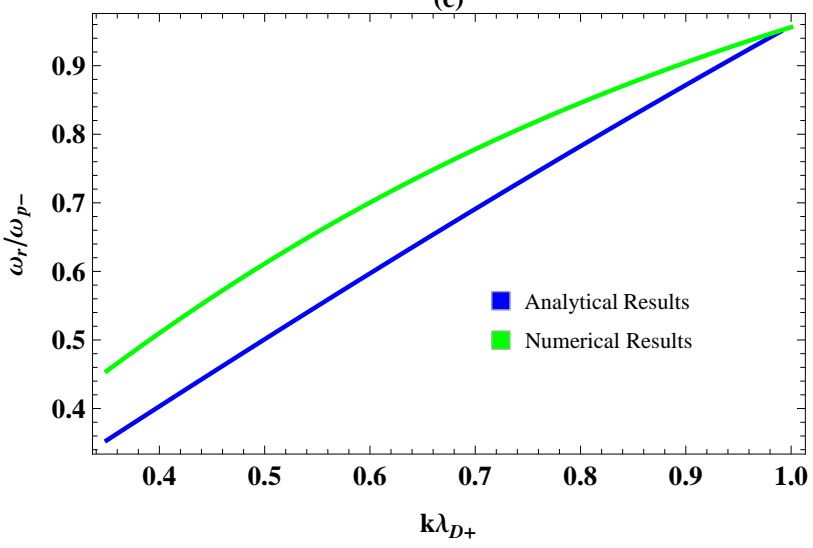

FIG. 3. The plots of normalized real frequency $\omega_{r} / \omega_{p_{-}}$ obtained (a)Analytically and (b)Numerically are presented against the normalized Debye length of positive ions $k \lambda_{D+}$, for the Kappa-distributed acoustic-laden twisted ion modes (c) A comparative plot between analytical (Blue) and numerical (Green) results at $\kappa_{+}=2, \kappa_{-}=3, m_{+} / m_{-}=0.001$ and $T_{+} / T_{-}=30$.

the temperature ratio of positive to negative ions $T_{+} / T_{-}$. This demeanor of normalized real frequency $\omega_{r} / \omega_{p_{-}}$is very lucid. Evidently, the large amount of energy is absorbed by the particles from the wave, which results in the enhancement of particles temperature and reduction in the magnitude of the normalized real frequency 
(a)

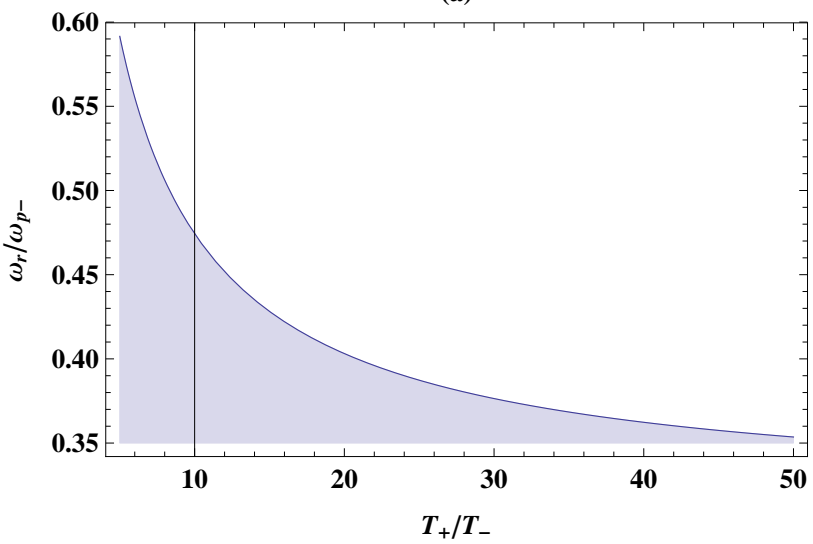

(b)
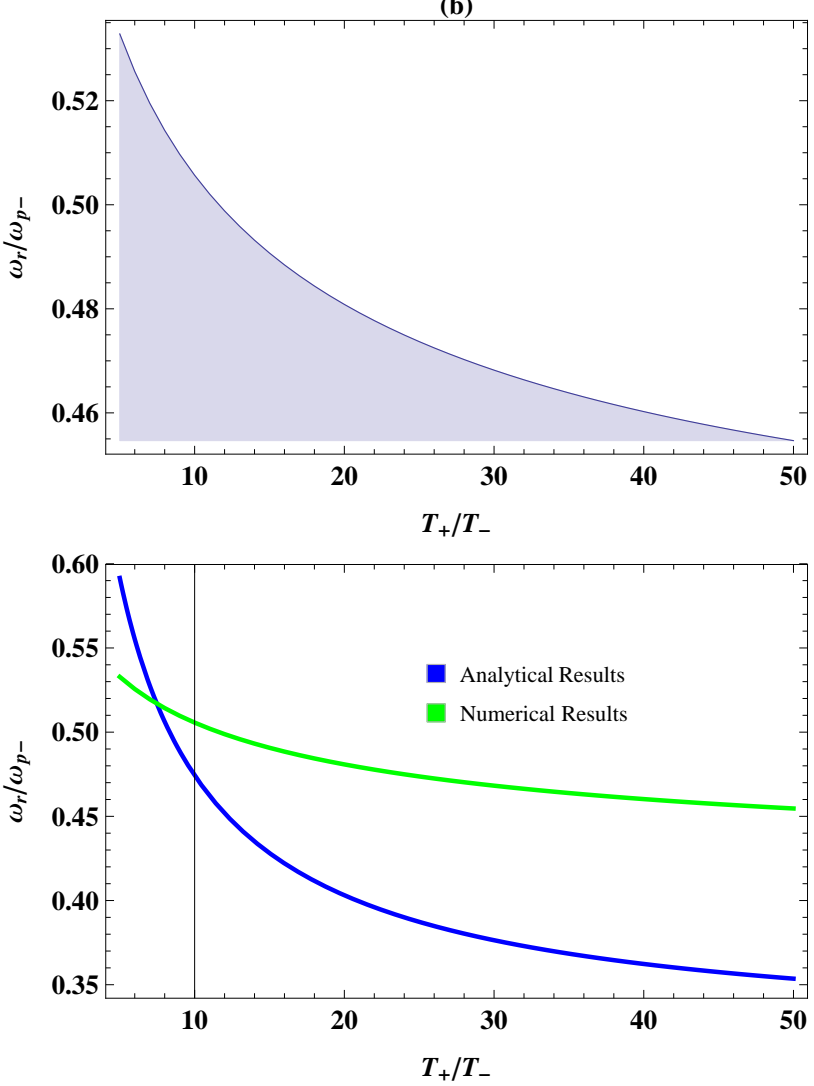

FIG. 4. The plots of normalized real frequency $\omega_{r} / \omega_{p_{-}}$ obtained (a)Analytically and (b)Numerically are presented against the temperature ratio of positive to negative ions $T_{+} / T_{-}$, for the Kappa-distributed acoustic-laden twisted ion modes (c) A contrast between Blue-analytical and Greennumerical curves at $\kappa_{+}=2, \kappa_{-}=3, m_{+} / m_{-}=0.001$ and $\varpi=0.5$.

$\omega_{r} / \omega_{p_{-}}$.

Figs. 4(a) and 4(b) show the analytical and numerical plots in the upper and lower panels respectively. The analytical plot is obtained from the approximated solution of the plasma dielectric function. But the exact numerical solution of the plasma dielectric function is utilized for the numerical plot. The analytical plot has reduced

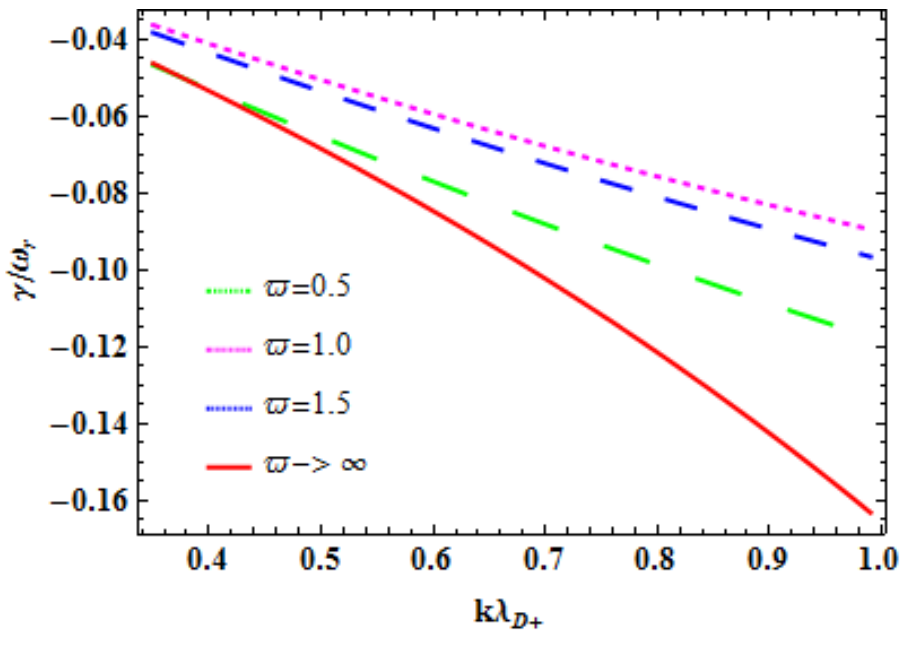

FIG. 5. The plots of normalized Landau damping rate $\gamma / \omega_{r}$ obtained analytically are presented against the normalized Debye length of positive ions $k \lambda_{D+}$, for the Kappa-distributed acoustic-laden twisted ion modes using different values of $\varpi=$ $k / l q_{\theta}$ at $\kappa_{+}=2, \kappa_{-}=3, m_{+} / m_{-}=0.001$ and $T_{+} / T_{-}=30$.

magnitude in comparison to the numerical plot.

The contrast between the analytical and numerical curves of the normalized real frequency $\omega_{r} / \omega_{p_{-}}$has been analyzed in the Fig. 4(c). Again, the frequency curves of the analytical and numerical solutions converge in the high frequency region, until they cross each other about $T_{+} / T_{-}=6$. On the other hand, the plot divulge deviation between approximative analytical and exact numerical curves in the low frequency region. The reason for this discrepancy is the endorsement of the essential approximation for computing the analytical results, whose validity is questionable in the low frequency region.

Instantly, we consider the acoustic-laden twisted ion waves in the non-Maxwellian Kappa-distributed asymmetric bi-ion plasma (having positive and negative ions with distinct masses and spectral indices) i.e., $m_{+} / m_{-}=$ $0.1, \kappa_{+}=2$ and $\kappa_{-}=3$. Figure 5 provides an illustration of numerical evaluations of the normalized Landau damping rate $\gamma / \omega_{r}$ which are plotted against the normalized Debye length of positive ions $k \lambda_{D+}$. Various values of the azimuthal wave number $l q_{\theta}$ have been considered. In fact, we have considered three cases of $\varpi=k / l q_{\theta}$ (the ratio of the parallel to the azimuthal wave number) to plot the normalized damping rate of the wave versus $k \lambda_{D+}$, namely (i) for the case with an azimuthal wave number $l q_{\theta}$ greater than the parallel wave number $k$ such that $\varpi=0.5$; (ii) the case when the azimuthal wave number $l q_{\theta}$ is equal to the parallel wave number $k$ such that $\varpi=1$; and (iii) for the case where the azimuthal wave number $l q_{\theta}$ is less than the parallel wave number $k$ such that $\varpi=1.5$, for the non-planar (azimuthal electric field) cases, respectively, and one for the planar electric field case (iv) for a very small azimuthal wave number (so that $l q_{\theta} \cong 0$ ) in comparison to the parallel wave number $k$ such that $\varpi=\infty$. 


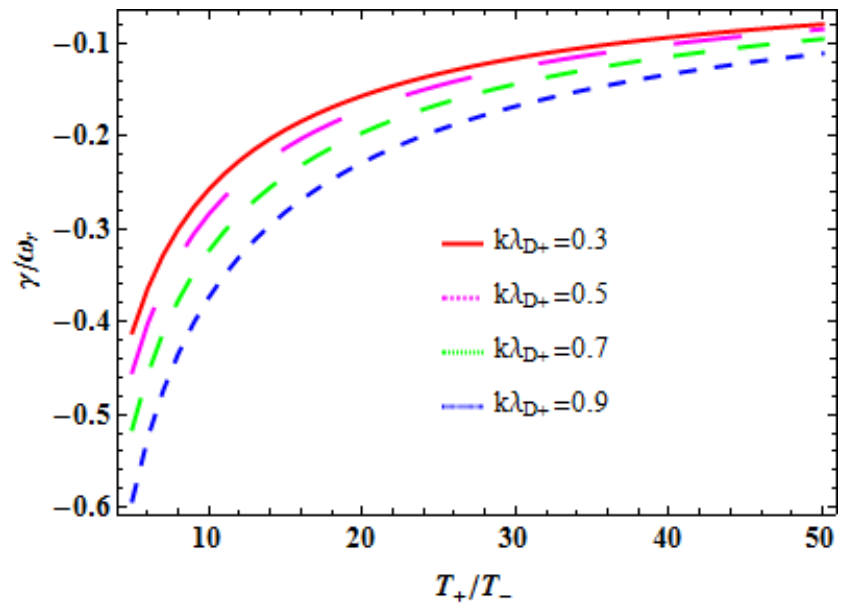

FIG. 6. The plots of the normalized Landau damping rate $\gamma / \omega_{r}$ are illustrated numerically against the temperature ratio of the positive to negative ions $T_{+} / T_{-}$, for the Kappadistributed acoustic-laden twisted ion modes using different values of $k \lambda_{D+}$ at $\kappa_{+}=2, \kappa_{-}=3, m_{+} / m_{-}=0.001$ and $\varpi=0.5$.

It can be observed from Figure 5 that the normalized Landau damping rate of the acoustic-laden twisted ion wave $\gamma / \omega_{r}$ is the smallest (in absolute value) for $\varpi=$ 1 , while it is slightly larger for the larger $\varpi=1.5$ and substantially larger for the smaller $\varpi=0.5$, as compared to each other for the non-planar (azimuthal electric field) cases. The physical reason for the wave damping rate to be so large for the $\varpi=0.5<1$ case seems to be that for large azimuthal wave numbers a larger number of particles will take energy from the wave so that the waveparticle interaction increases. Therefore, the damping rate of the wave for the $\varpi=0.5<1$ case is traced larger (in absolute value) than for the cases $\varpi=1.5$ and $\varpi=1$. However, if we compare the wave damping rate of the twisted wave cases $(\varpi=0.5,1,1.5)$ with the planar case $(\varpi=\infty)$, the red planar curve appears below the twisted wave curves in the figure, thus showing higher damping rate values for the planar mode in comparison to non-planar acoustic-laden (twisted) ion mode.

The exact numerical results are demonstrated for the acoustic-laden twisted ion waves in Figure 6. Again, we have non-Maxwellian Kappa-distributed asymmetric biion plasma (having positive and negative ions with distinct masses and spectral indices), i.e. $m_{+} / m_{-}=0.1$, $\kappa_{+}=2$ and $\kappa_{-}=3$. Plots of the normalized Landau damping rate $\gamma / \omega_{r}$ are shown and analyzed against the temperature ratio of the positive to negative ions $T_{+} / T_{-}$. Actually, four discrete values of the normalized Debye length of the positive ions $k \lambda_{D+}$ are chosen to investigate the normalized Landau damping rate $\gamma / \omega_{p_{-}}$of the acoustic-laden twisted ion waves (viz. $k \lambda_{D+}=0.3$, $\left.k \lambda_{D+}=0.5, k \lambda_{D+}=0.7 \& k \lambda_{D+}=0.9\right)$. It is revealed that the Landau damping rate $\gamma / \omega_{p_{-}}$is minimal for the lower value of the normalized Debye length of the positive ions such that $k \lambda_{D+}=0.3$, while the Landau damping rate $\gamma / \omega_{r}$ is maximal with $k \lambda_{D+}=0.9$. It is obvious that the larger value of $k \lambda_{D+}$ corresponds to large number of probable supra-thermal particles, taking energy from the wave through the wave-particle interaction. That is why the Landau damping rate $\gamma / \omega_{r}$ of the blue curve is maximal. On the other hand, the number of supra-thermal particles participating in the wave-interaction phenomena are reduced with the smaller value of $k \lambda_{D+}$, which corresponds to the minimal damping of the acousticladen twisted ion waves.

Figure 7 involves both (a)analytical and (b)an exact numerical exhibition of the acoustic-laden twisted ion waves in the non-Maxwellian Kappa-distributed asymmetric bi-ion plasma (having positive and negative ions with distinct masses and spectral indices), i.e. $m_{+} / m_{-}=$ $0.1, \kappa_{+}=2$ and $\kappa_{-}=3$. The normalized Landau damping rate $\gamma / \omega_{r}$ is examined against the temperature ratio of the positive to negative ions $T_{+} / T_{-}$. The azimuthal wave number $l q_{\theta}$ has been assigned three values correlating the non-planar morphology of the electric field. The three non-planar cases of $\varpi=k / l q_{\theta}$ (the ratio of the parallel to the azimuthal wave number) are inspected, specifically (i) for the case with the parallel wave number $k$ less than an azimuthal wave number $l q_{\theta}$ such that $\varpi=0.5$; (ii) the case when the parallel wave number $k$ is equal to the azimuthal wave number $l q_{\theta}$ such that $\varpi=1$; and (iii) for the case where the parallel wave number $k$ is greater than the azimuthal wave number $l q_{\theta}$ such that $\varpi=1.5$, for the non-planar (azimuthal electric field) cases, respectively. It is evident that the normalized Landau damping rate of the acoustic-laden twisted ion wave $\gamma / \omega_{r}$ is least (in absolute value) for $k / l q_{\theta}=1$, while it is marginally larger for the larger $k / l q_{\theta}=1.5$ and essentially larger for the smaller $k / l q_{\theta}=0.5$, as compared to each other for the twisted (azimuthal electric field) cases. Physically, the large number of particles are absorbing energy from the acoustic-laden twisted ion wave for the small value of $\varpi=0.5<1$ in comparison to the $\varpi=1.5>1$ and $\varpi=1.0$. Therefore, the Landau damping rate of the wave for the $\varpi=0.5<1$ case is traced below the $\varpi=1.5$ and $\varpi=1.0$ cases.

Figure $7(\mathrm{a})$ presents the approximated analytical curves of the normalized Landau damping rate $\gamma / \omega_{r}$ of the acoustic-laden twisted ion wave, while Fig. 7(b) illustrates the numerical results obtained from the exact numerical solution of the plasma dielectric function of the Kappa-distributed asymmetric bi-ion plasma.

The contrast of the panels (a) Analytical results \& (b) Numerical results has been depicted in Fig. 7(c). The analytical and exact numerical curves elevate almost parallel in the weak damping region. The minor difference appears between the analytical and numerical curves because we have expanded the plasma dispersion function to the second order term. In the strong damping region, the analytical curves start rising upward about $T_{+} / T_{=} 4$, which contradicts the physical interpretation of the Landau damping rate. But the exact numerical results keep the true interpretation of the Landau damping rate even 
(a)

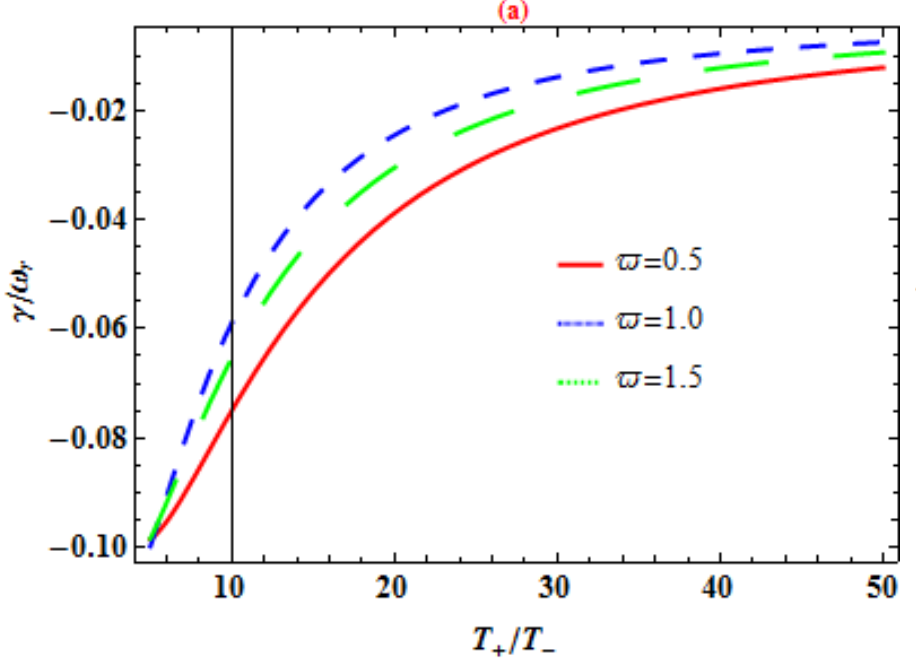

(b)

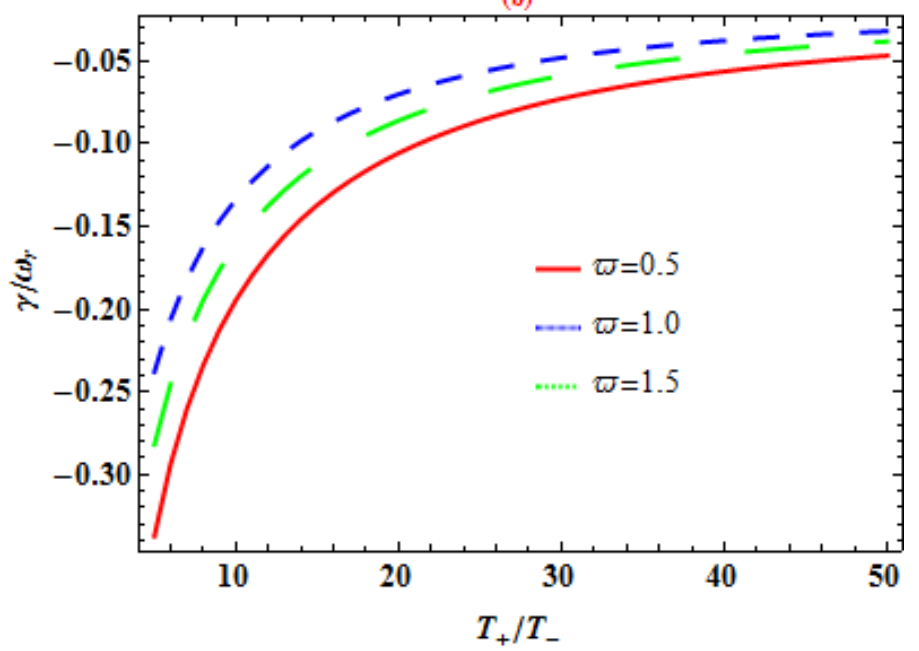

(c)

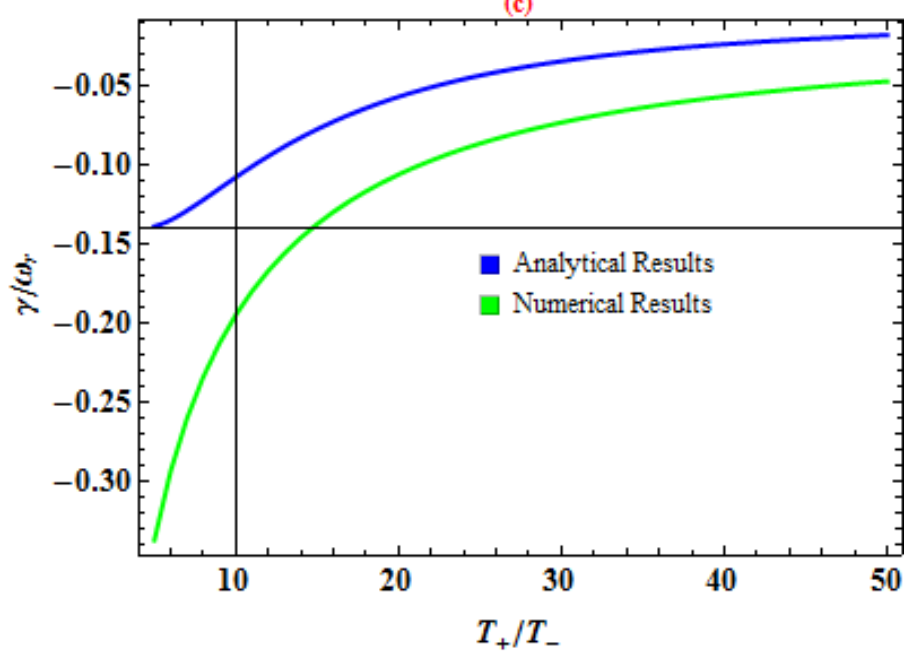

(a)

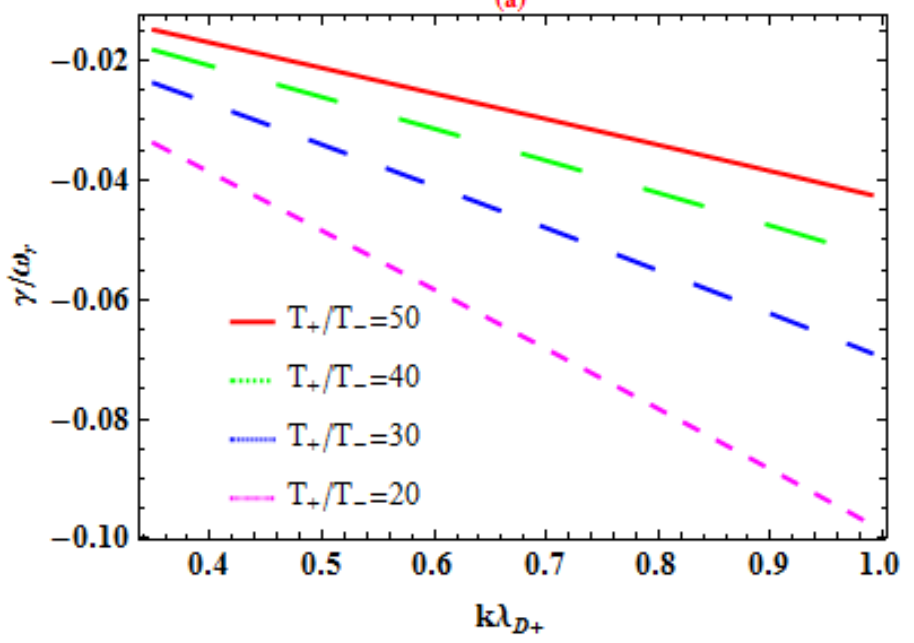

(b)

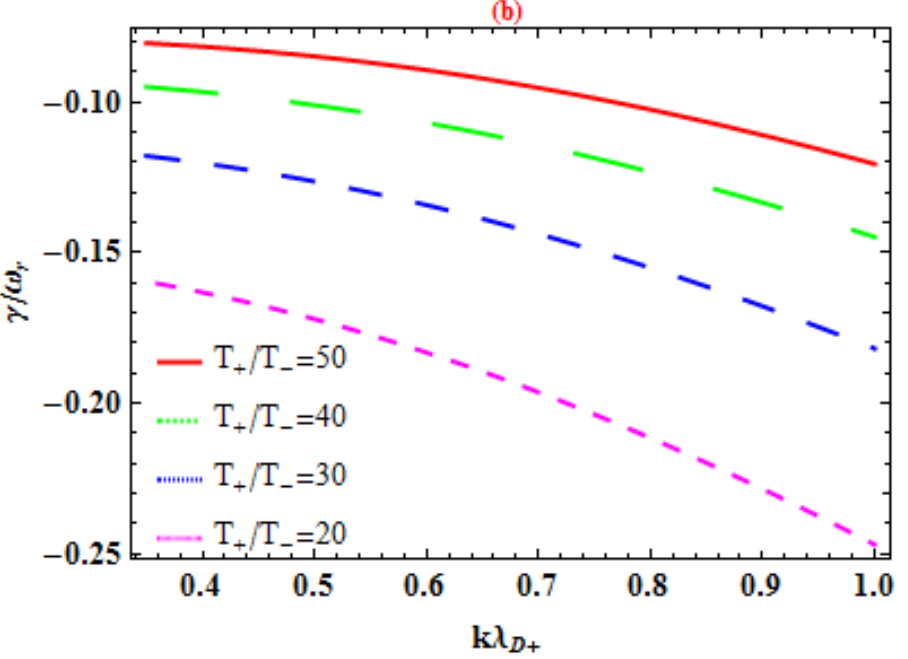

(c)

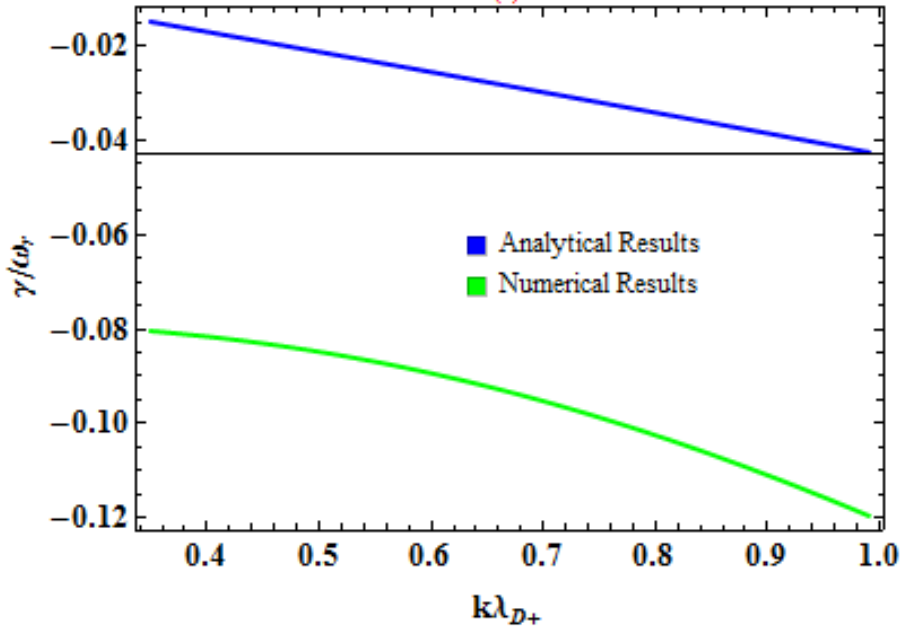

FIG. 7. The plots of the normalized Landau damping rate $\gamma / \omega_{r}$ are obtained (a)Analytically and (b)Numerically against the temperature ratio of the positive to negative ions $T_{+} / T_{-}$, for the Kappa-distributed acoustic-laden twisted ion modes using different values of $\varpi$ (c) Distinctive curves of analytical and numerical results of the Kappa-distributed asymmetric bi-ion plasma for $\varpi=0.5$ at $\kappa_{+}=2, \kappa_{-}=3, m_{+} / m_{-}=0.001$ and $k \lambda_{D+}=0.5$.
FIG. 8. The plots of normalized Landau damping rate $\gamma / \omega_{r}$ obtained (a)Analytically and (b)Numerically are presented against the normalized Debye length of positive ions $k \lambda_{D+}$, for the Kappa-distributed acoustic-laden twisted ion modes using different values of $T_{+} / T_{-}$(c) Disparity between the analytical and numerical results of the Kappa-distributed asymmetric bi-ion plasma for $T_{+} / T_{-}=50$ at $\kappa_{+}=2, \kappa_{-}=3$, $m_{+} / m_{-}=0.001$ and $\varpi=0.5$. 
in the strong damping region and follow the same downward trend of the Landau damping rate.

Here, we consider the case of non-Maxwellian Kappadistributed asymmetric bi-ion plasma, namely with $\kappa_{+}=$ $2 ; \kappa_{-}=3$, and such that the temperature of the positive ions is greater than that of the negative ions i.e., $T_{+}>T_{-}$. Figure 6 illustrates (a)analytical and (b) exact numerical evaluations of the normalized Landau damping rates $\gamma / \omega_{r}$, plotted against the normalized Debye length of the positive ions, $k \lambda_{D+}$, of the acousticladen twisted ion waves for various values of the temperature ratio of positive to negative ions $T_{+} / T_{-}$. We have chosen four different temperature ratios of positive to negative ions, viz. $T_{+} / T_{-}=20,30,40$, and 50 , to illustrate the effect of this temperature ratio. It can be observed that $\gamma / \omega_{r}$ is minimal for $T_{+} / T_{-}=50$ (corresponding to maximal damping rate $\gamma / \omega_{r}$ ) and maximal for $T_{+} / T_{-}=20$ (corresponding to minimal damping rate). This analysis thus clearly reveals that the Landau damping rate of the acoustic-type twisted ion waves increases when the temperature ratio of positive to negative ions $T_{+} / T_{-}$decreases (as can be seen in Fig. 6). The reason for such behavior is again associated with the waveparticle interaction phenomenon. If the temperature ratio of positive to negative ions $T_{+} / T_{-}$is higher, then the suprathermal particles can provide more energy to the wave and the Landau damping rate of the acoustic-laden twisted ion wave decreases. Hence, the Landau damping rate is larger for the smaller value of the temperature ratio of positive to negative ions $T_{+} / T_{-}$, because the energy supply consequently reduces with lower $T_{+} / T_{-}$.

Figure 8(a) demonstrates the approximated analytical curves of the normalized Landau damping rate $\gamma / \omega_{r}$ of the acoustic-laden twisted ion waves. On the other hand, Fig. 8(b) illustrates the exact numerical results of the acoustic-laden twisted ion waves, which are accomplished from the approximation-free solution of the plasma dielectric function of asymmetric bi-ion plasma.

Fig. 8(c) represents the correlation between the damping rates $\gamma / \omega_{r}$ of the analytical and numerical results. The damping curves of the analytical curve are more steeper and have weak Landau damping rate $\gamma / \omega_{r}$ in comparison to the numerical curve. While the damping rate of the numerical results remain good even in the strong damping region. This is because that analytical results are approximated and obtained from the expansion of the plasma dispersion function up to the square term by employing the resonance conditions of positive and negative ions. While, the numerical results are achieved from the exact solution of the plasma dielectric function without any approximation.

Figure 9 presents the numerical plots of normalized Landau damping rates $\gamma / \omega_{r}$ versus the normalized Debye length of positive ions $k \lambda_{D+}$ of the acoustic-laden twisted ion waves at various values of the spectral indices $\kappa_{+}$and $\kappa_{-}$. We have selected three cases with distinct and one cases with similar spectral indices. For the distinct Kappa-distributed i.e., $\kappa_{+}=2$ and $\kappa_{-}=3$ and

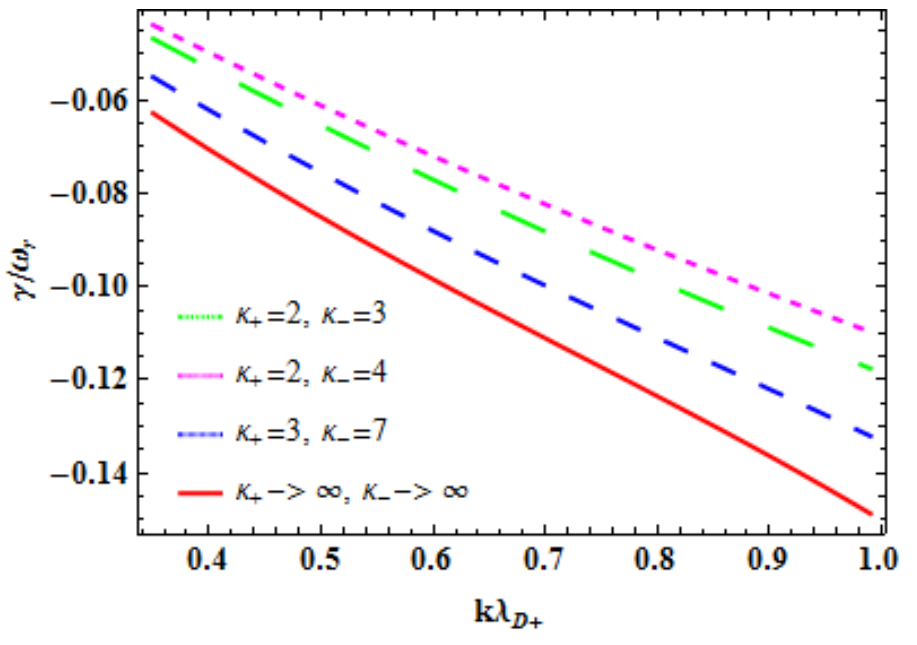

FIG. 9. The plots of normalized Landau damping rate $\gamma / \omega_{r}$ obtained analytically are presented against the normalized Debye length of positive ions $k \lambda_{D+}$, for the Kappa-distributed acoustic-laden twisted ion modes using different values of spectral indices $\kappa_{+}$and $\kappa_{-}$at $\varpi=0.5, m_{+} / m_{-}=0.001$ and $T_{+} / T_{-}=30$.

Maxwellian distributed i.e., $\kappa_{+}=\kappa_{-} \rightarrow \infty$ spectral indices, the Landau damping rate of acoustic-laden twisted ion waves is higher for the Kappa-distributed spectral indices in comparison to the Maxwellian distributed case. The physical reason for this fact is the presence of the large number of supra-thermal particles for small values of the spectral index, which results in the absorption of more energy from the wave as compare to Maxwellian distributed particles. That is why the damping rate of the acoustic-type twisted ion waves in bi-ion plasma is larger in Kappa-distributed plasma as compare to Maxwellian plasma. For the distinct spectral indices, the Landau damping rate of the acoustic-laden twisted ion waves in larger for the $\kappa_{+}<\kappa_{-}$and smaller for the case where $\kappa_{+}>\kappa_{-}$.

In Figure 10, a colored contour plot is shown of the normalized damping rate $\gamma / \omega_{r}$ of the acoustic-laden twisted ion waves in non-Maxwellian Kappa-distributed asymmetric bi-ion plasma as a function of the normalized Debye length of the positive ions $k \lambda_{D+}$ and $\varpi=k / l q_{\theta}$, the ratio of the parallel to the azimuthal wave number with $T_{+} / T_{-}=30$. It shows that for increasing values of $\varpi=k / l q_{\theta}$ (and a fixed value of $k \lambda_{D+}$ ), the magnitude of the damping rate of the wave $\left(\left|\gamma / \omega_{r}\right|\right)$ decreases. The same trend in the damping rate also occurs for a fixed value of $\varpi=k / l q_{\theta}$ and a decreasing value of $k \lambda_{D+}$. The large values of $k \lambda_{D+}$ correspond to higher spatial dimensions and, consequently, the presence of large numbers of suprathermal particles in the energy distribution spectrum. When enormous amounts of energetic particles interact with the wave, the magnitude of the absorbedenergy enhances by the wave-particle interaction phenomena. Hence, the particles take more energy from the wave and this yields higher Landau damping rates of the 


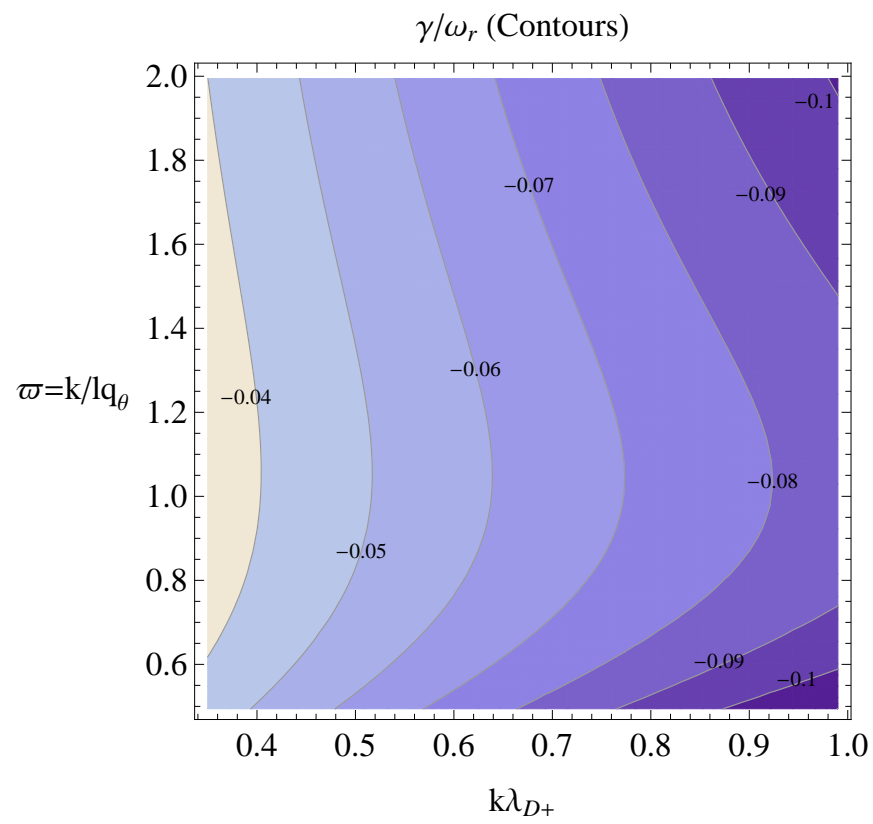

FIG. 10. Colored contour plots of normalized Landau damping rate $\gamma / \omega_{r}$ obtained analytically are presented against the normalized Debye length of positive ions $k \lambda_{D+}$ and $\varpi=$ $k / l q_{\theta}$, for the Kappa-distributed acoustic-laden twisted ion modes at $\kappa_{+}=2, \kappa_{-}=3, m_{+} / m_{-}=0.001$ and $T_{+} / T_{-}=30$.

acoustic-laden twisted ion wave and vice versa.

Figure $\mathbf{1 1}$ is a colored contour plot of the normalized Landau damping rate $\gamma / \omega_{r}$ of the Kappa-distributed acoustic-laden twisted ion waves as function of the temperature ratio of positive to negative ions $T_{+} / T_{-}$and the ratio of the conventional to the azimuthal wave number $\varpi$ in an asymmetric bi-ion (BI) plasma with $k \lambda_{D+}=0.3$. It can be seen that the magnitude of the Landau damping rate of the Kappa-distributed acoustic-laden twisted ion waves $\left|\gamma / \omega_{r}\right|$ decreases with increasing values of $\varpi$ (i.e., when the azimuthal wave number $l q_{\theta}$ is smaller as compare to the conventional wave number $k$ ). The effect of the ratio of the temperature of positive to that of negative ions, $T_{+} / T_{-}$, on the damping rate of the wave is also clarified. The figure reveals that the magnitude of the Landau damping rate $\left|\gamma / \omega_{r}\right|$ shows an decreasing trend with increasing values of the ratio $T_{+} / T_{-}$, which means that the temperature of the bi-ion plasma may be due to the energy injected by suprathermal particles. When the particles absorb more energy from the wave the Landau damping rate is smaller, and when the particles take less energy from the wave, the damping is larger.

Figure 12 is a colored contour plot of the normalized Landau damping rate $\gamma / \omega_{r}$ of the Kappa-distributed acoustic-laden twisted ion waves versus the normalized Debye length of positive ions $k \lambda_{D+}$ and the mass ratio of positive to negative ions $m_{+} / m_{-}$in an asymmetric Kappa-distributed bi-ion (BI) plasma with $T_{+} / T_{-}=30$ and $\varpi=k / l q_{\theta}=0.5$. It is shown that the magnitude of the Landau damping rate $\left|\gamma / \omega_{r}\right|$ of the wave increases

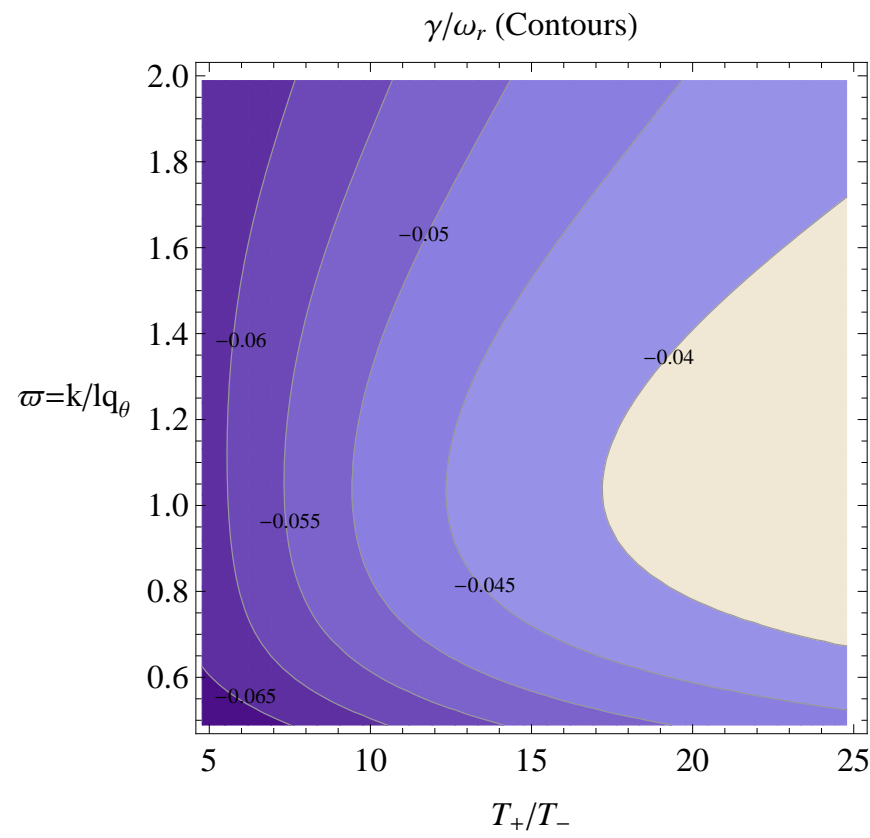

FIG. 11. Colored contour plots of normalized Landau damping rate $\gamma / \omega_{r}$ obtained analytically are presented against the temperature ratio of positive to negative ions $T_{+} / T_{-}$ and $\varpi=k / l q_{\theta}$, for the Kappa-distributed acoustic-laden twisted ion modes at $\kappa_{+}=2, \kappa_{-}=3, k \lambda_{D+}=0.3$ and $m_{+} / m_{-}=0.001$.

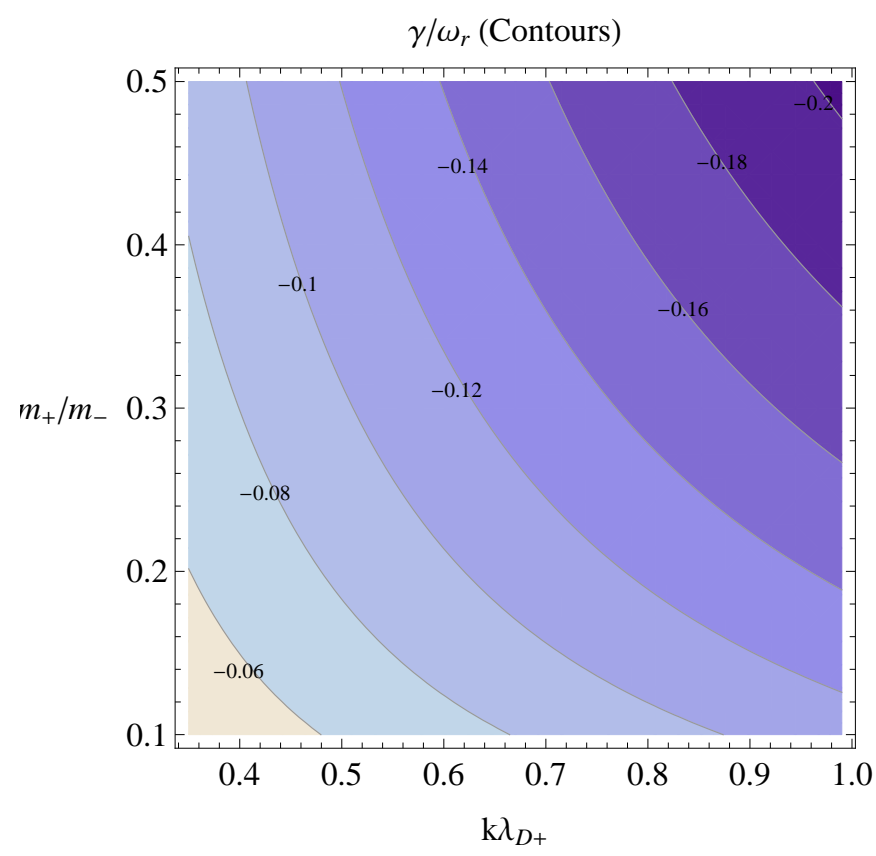

FIG. 12. The contour plots of normalized Landau damping rate $\gamma / \omega_{r}$ obtained analytically are presented against the normalized Debye length of positive ions $k \lambda_{D+}$ and mass ratio $m_{+} / m_{-}$, for the Kappa-distributed acoustic-laden twisted ion modes at $\kappa_{+}=2, \kappa_{-}=3, \varpi=k / l q_{\theta}=0.5$ and $T_{+} / T_{-}=30$. 
with increasing dimensionless Debye length $k \lambda_{D+}$ of positive ions. This can be understood as follows: with larger Debye length $k \lambda_{D+}$, the wave finds a larger number of probable particles that take energy from the wave to enhance its damping in comparison to the case with a shorter Debye length $k \lambda_{D+}$. It is observed that, as the mass of positive ion $m_{+}$approaches the mass of the negative ions $m_{-}$, i.e. as $m_{+} / m_{-} \rightarrow 1$, the damping rate of the wave increases. This is due to the fact that the increasing asymmetry in the mass of Kappa-distributed bi-ion plasma lowers the number of particles taking energy of the wave. That is why the heavy negative ions have a higher magnitude of the damping rate in comparison to positive ions.

\section{CONCLUSIONS}

To conclude, in the present paper, a twisted kinetic model has been developed to estimate the weak Landau damping rates with mass-symmetry for both Kappa and Maxwellian distributed bi-ion plasmas. The traditional ion waves with twisting character are detected, at least as long as the symmetry of the bi-ion plasmas remain invariant. The twisting is incubated by the phase variation of the non-planar helical wave-fronts along the spatial and azimuthal dimensions. The delicate doping of high mass immobile ions may cause a broken-symmetry, and then we have asymmetric bi-ion plasma, which exhibits the novel acoustic-laden twisted ion waves. The set of Vlasov-Poisson equations have been solved under the paraxial approximation to manipulate the decomposed distribution function and potential via the Laguerre Gaussian (LG) mode function. The numerical evaluations of the obtained weak damping rate expressions are also illustrated for the peculiar acoustic-laden twisted ion waves in the asymmetric case of the Kappadistributed bi-ion plasmas. The weak and strong damping results have been analyzed to show its dependency on various parameters, like the normalized wave number $k \lambda_{D+}$, the axial to azimuthal wave number ratio $\varpi=k / l q_{\theta}$, the spectral indices $\kappa_{+} \& \kappa_{-}$, the positive to negative ion temperature ratio $T_{+} / T_{-}$and the mass ratio $m_{+} / m_{-}$.

The weak Landau damping rates of the planar ion waves (i.e. $l q_{\theta} \cong 0$ ) are higher in comparison to the twisted ion waves (i.e., $k<l q_{\theta}, k=l q_{\theta} \& k>l q_{\theta}$ ). For the twisted cases, the damping is enhanced for $k<l q_{\theta}$, reduced when $k>l q_{\theta}$ and least depreciated with $k=l q_{\theta}$. The twisted ion waves are significantly damped for the smaller values of the positive to negative ion temperature ratio $T_{+} / T_{-}$and a reduced damping is attained for the larger positive to negative ion temperature ratio $T_{+} / T_{-}$.

The normalized real frequency $\omega_{r} / \omega_{p_{+}}$of the twisted acoustic-laden ion waves is found to be growing with the normalized Debye length of the positive ions $k \lambda_{D+}$ and falling relative to the positive to negative ion temperature ratio $T_{+} / T_{-}$. The numerical plots show higher magnitude of the real frequency in comparison to the analytical plots.

The weak Landau damping rates of the twisted acoustic-laden ion waves are minimal for $k=l q_{\theta}$, larger for $k<l q_{\theta}$ and smaller for $k>l q_{\theta}$. For the planar case of the acoustic-type ion wave, i.e. $l q_{\theta} \cong 0$, the Landau damping rates are the highest. The twisted acousticladen ion waves are highly damped for smaller values of the positive to negative ion temperature ratio $T_{+} / T_{-}$and a declined damping curve is obtained for larger positive to negative ion temperature ratio $T_{+} / T_{-}$. The damping curves are also examined for the distinct spectral indices of positive and negative ions, i.e. $\kappa_{+} \& \kappa_{-}$. The twisted acoustic-laden ion waves are weakly damped for smaller values of the spectral indices and the damping rate increases as the value of Kappa index approaches the Maxwellian limit. The results of the investigations are further clarified with contour plots. The ascending damping magnitudes are depicted with rising normalized wave number $k \lambda_{D+}$, descending axial to azimuthal wave number ratio $\varpi=k / l q_{\theta}$, depreciation in the fraction of positive to negative ion temperature $T_{+} / T_{-}$and reduced mass ratio of positive to negative ions,$m_{+} / m_{-}$.

The strong damping results of the acoustic-laden twisted ion waves are achieved from the exact solution of the plasma dispersion function by employing standard Newton-Raphson method. In particular, the strong damping curves are attained to examine the authentic demeanor of the normalized Landau damping rates $\gamma / \omega_{r}$ in the strong damping region. The damping of the twisted acoustic-laden ion wave is more stronger for the broader range of the normalized wave number i.e., $k \lambda_{D+}=0.9$ and less stronger for the relatively shorter range i.e., $k \lambda_{D+}=0.3$. The strong damping rates of the acousticladen twisted ion waves are maximal for the $k<l q_{\theta}$, smaller for $k>l q_{\theta}$ and least for $k=l q_{\theta}$. The twisted acoustic-laden ion waves have higher magnitude of the damping rate for smaller values of the positive to negative ion temperature ratio $T_{+} / T_{-}$and a reduced magnitude of the damping curve is obtained for larger positive to negative ion temperature ratio $T_{+} / T_{-}$.

The asymmetry can originate from many neoteric phenomena in plasmas, like the existence of acoustic-laden twisted ion wave in Kappa-distributed asymmetric bi-ion plasmas. The mass of the bi-ions (positive and negative ions) does not remain similar under a broken-symmetry. On the contrary, the adhesion/cohesion of the high-mass immobile ions may constitute heavier negative ions and lighter positive ions in the bi-ion plasmas. The corresponding underweight positive ions and massive negative ions reinforce the acoustic signatures in the Kappadistributed bi-ion plasmas. Such mass oriented asymmetries may appear in electron-positron plasmas, electron hole plasmas, astrophysical jets, pulsar magnetospheres and dusty plasmas.

We have also introduced the non-planar (azimuthal) essence of the Kappa-distributed bi-ion plasma. The azimuthal parameter has been incorporated by consider- 
ing the exchange of orbital angular momentum between the helical field and the plasma modes. This deportation persuades the non-planar morphology of the wavefronts, which is comprised by the propagation of the wave along the axial and azimuthal directions. The discovered morphologies of the astrophysical and space plasmas are non-planar like ring-shaped, helical, spiral, etc. Therefore, it is reasonable to implicate the azimuthal factor ascribing the phase variation relative to geometrical morphology. Remark that we have selected the non-Maxwellian or Kappa aspect of the plasma species, i.e. positive and negative ions. The adequate distribution of the inertial (heavier) particles is customarily adopted as Maxwellian, if the inertialess particles are non-Maxwellian distributed. However, the distribution of the bi-ions has been defined as Lorentzian or Kappa distribution, but the Kappa index of the massive species is larger correlative to lighter species. Massive particles like protons, ions, and dust particles are certainly cooler, and accordingly their distributions must be Maxwellian as for inertialess particles. In space plasmas, the distributions of inertialess species, like electrons, is wellreproduced by a Kappa-distributed. Hence, it is acceptable to assume a larger spectral index for the heavier particles correlated lighter particles. Twisted waves can significantly contribute to the current understanding of the asymmetric plasmas. These studies may devise many concealed features of the non-Maxwellian plasmas especially correlative to ion acoustic waves. In the future, we can discover a collection of new twisted and planar modes by meditating various asymmetries in space and laboratory plasmas.

\section{ACKNOWLEDGMENT}

KA acknowledges the Centre for mathematical Plasma Astrophysics (CmPA) to support the study of twisted waves at Department of Mathematics, KU Leuven, Belgium. These results were obtained in the framework of the projects C14/19/089 (C1 project Internal Funds KU Leuven), G.0D07.19N (FWO-Vlaanderen), C 90347 (ESA Prodex) and Belspo BRAIN project BR/165/A2/CCSOM.

\section{DATA AVAILABILITY}

The data/parameters that supports the findings of this study are available within the article [Kashif Arshad, Yana G. Maneva, and S. Poedts (2017), "Ion acoustic wave damping in a non-Maxwellian bi-ion electron plasma in the presence of dust", AIP Publishing, http://dx.doi.org/10.1063/1.4995581].

\footnotetext{
${ }^{1}$ K. Arshad and S. Mahmood, Phys. Plasmas 17, 124501 (2010).
}

${ }^{2}$ S. M. Mahajan and N. L. Shatashvili, Phys. Plasmas 15, 100701 (2008).

${ }^{3}$ S. M. Mahajan, N. L. Shatashvili, and V. I. Berezhiani, Phys. Rev. E 80, 066404 (2009).

${ }^{4}$ V. I. Berezhiani and S. M. Mahajan, Phys. Rev. Lett. 73, 1110 (1994).

${ }^{5}$ V. I. Berezhiani and S. M. Mahajan, Phys. Rev. E 52, 1968 (1995).

${ }^{6}$ A. Esfandyari-Kalejahi, I. Kourakis, M. Mehdipoor, and P. K. Shukla, J. Phys. A 39, 13817 (2006).

${ }^{7}$ G. G. Howes, Phys. Plasmas 25, 055501 (2018).

${ }^{8}$ K. Arshad, A. Rehman and S. Mahmood, Phys. Plasmas 22, 112114 (2015); Phys. Plasmas 23, 052107 (2016).

${ }^{9}$ K. Arshad, M. Lazar, S. Mahmood, A. Rehman and S. Poedts, Phys. Plasmas 24, 033701 (2017).

${ }^{10}$ S. Ali, S. Bukhari, and J. T. Mendonca, Phys. Plasmas 23, 033703 (2016); J. D. Rodrigues, H. Tercas, and J. T. Mendonca, Europhys. Lett. 113, 13001 (2016)

${ }^{11}$ Z. Ehsan, N. L. Tsintsadze, H. A. Shah, R. M. G. M. Trines and M. Imran, Phys. Plasmas 23, 062125 (2016).

${ }^{12}$ K. Arshad, Y. G. Maneva and S. Poedts, Phys. Plasmas 24, 093708 (2017).

${ }^{13}$ S. Wedemeyer, E. Scullion, O. Steiner, J. de la Cruz Rodriguez, L. R. vander Voort, J. Phys. Conference Series 440, 012005 (2013); P. K. Shukla, J. Geophys. Research: Space Phy. 118, 1 (2013).

${ }^{14}$ Iwo Bialynicki-Birula, Phys. Rev. Lett. 118, 114801 (2017); S. M. Barnett, Phys. Rev. Lett 118, 114802 (2017).

${ }^{15}$ J. Vieira, R. M. G. M. Trines, E. P. Alves, R. A. Fonseca, J. T. Mendonca, R. Bingham, P. Norreys and L.O. Silva, Nature Commun. 7, 10371 (2016).

${ }^{16}$ A. Jesacher, S. Furhapter, S. Bernet, and M. Ritsch-Marte, Phys. Rev. Lett. 94, 233902 (2005); B. Jack, J. Leach, J. Romero, and M. J. Padgett, Phys. Rev. Lett. 103, 083602 (2009).

${ }^{17}$ J. Wang, J. Yang, I. M. Fazal, N. Ahmed, Y. Yan, H. Huang, Y. Ren, Y. Yue, S. Dolinar, M. Tu, and A. E. Willner, Nat. Photonics 6, 488 (2012).

${ }^{18}$ I. Bialynicki-Birula and Z. Bialynicka-Birula, New J. Phys. 18, 023022 (2016).

${ }^{19}$ B. Zolesi and L. R. Cander, Ionospheric Prediction and Forecasting, Springer Geophysics Springer-Verlag, Berlin, Hiedelberg, (2014).

${ }^{20}$ S. Oemrawsingh, X. Ma, D. Voigt, A. Aiello, E. Eliel, G.t Hooft, and J. P. Woerdman, Phys. Rev. Lett. 95, 240501 (2005).

${ }^{21}$ J. T. Mendonca, F Haas and A.Gammal, J. Phys. B 49, 14 (2016).

${ }^{22}$ F. Tamburini, B. Thide, G. Molina-Terriza, and G. Anzolin, Nat. Phys. 7, 195 (2011).

${ }^{23}$ S. A. Khan, A. Rehman and J.T. Mendonca, Phys. Plasmas 21, 092109 (2014).

${ }^{24}$ K. Arshad, S. Mahmood and A. M. Mirza, Phys. Plasmas 18, 092115 (2011); K. Arshad and A. M. Mirza, Astrophys. Space Sci. 349, 753 (2014); K. Arshad, A. M. Mirza and A. Rehman, Astrophys. Space Sci.350, 585 (2014); K. Arshad, F. Siddique, A. M. Mirza and A. Rehman, Astrophys. Space Sci. 350, 169 (2014); K. Arshad, Z. Ehsan, S. A. Khan and S. Mahmood, Phys. Plasma 21, 023704 (2014).

${ }^{25}$ K. Arshad, "Application of Kinetic Theory to Study Twisted Modes in non-Maxwellian Plasmas", Ph.D. thesis, Centre for Plasma-Astrophysics, KU Leuven, May (2018).

${ }^{26}$ A. F. Alexandrov, L. S. Bogdankevich and A. A. Rukhadze, "Principles of Plasma Electrodynamics", SpringerVerlag Berlin, Heidelberg, New York, 1984.

${ }^{27}$ D. A. Gurnett and A. Bhattacharjee, "Introduction to Plasma Physics: With Space and Laboratory Applications", Cambridge University Press (2005).

${ }^{28}$ P. Dennery and A. Krzywiki, "Mathematics for Physicists", Harper and Row, New York (1967). 